TRANSACTIONS OF THE

AMERICAN MATHEMATICAL SOCIETY

Volume 363, Number 7, July 2011, Pages 3769-3797

S 0002-9947(2011)05284-0

Article electronically published on February 16, 2011

\title{
TWO-PARAMETER QUANTUM VERTEX REPRESENTATIONS VIA FINITE GROUPS AND THE MCKAY CORRESPONDENCE
}

\author{
NAIHUAN JING AND HONGLIAN ZHANG
}

\begin{abstract}
We provide a group-theoretic realization of two-parameter quantum toroidal algebras using finite subgroups of $S L_{2}(\mathbb{C})$ via McKay correspondence. In particular our construction contains the vertex representation of the two-parameter quantum affine algebras of $A D E$ types as special subalgebras.
\end{abstract}

\section{INTRODUCTION}

In a series of papers FJW1, FJW2 the basic representations of two-toroidal Lie algebras and their quantum analogs, including affine Lie algebras and quantum affine algebras of simply laced types as subaglebras, were constructed from the representation theory of finite groups of $S L_{2}(\mathbb{C})$ via the celebrated McKay correspondence. Using purely representation-theoretic data the Frenkel-Kac [FK] and Frenkel-Jing [FJ] vertex representations are constructed as a by-product of the unified group-theoretic constructions from the root lattice of the corresponding finite-dimensional Lie algebra g. In [J4] we have pointed out that such a uniformed construction incorporates not only the toroidal Lie algebras and quantum toroidal algebras [VV] (see also [J3]) but also gives a general algebraic machinery to realize other related algebraic structures.

In the current paper we provide a two-parameter quantum analog of the toroidal Lie algebras of simply laced types using the new form of McKay correspondence. In particular this gives a group-theoretic realization of the newly revitalized twoparameter quantum affine algebras [HRZ, Z as distinguished subalgebras of our new quantum toroidal algebras. As expected, our construction degenerates to the quantum affine case by specializing $r=s^{-1}$. Through this new construction we have also shown that the vertex representation constructed in $[\mathrm{HZ}, \mathrm{Z}]$ is a natural generalization of the Frenkel-Jing construction and also reconfirms the two-parameter generalization of the usual quantum affine algebras and their Drinfeld realization. Our construction further shows that the toroidal version is more suitable and natural in this picture and reveals more symmetry in the structure of the two-parameter quantum toroidal algebras.

The new two-parameter quantum toroidal Lie algebras or double affine algebras provide a new layer of generalization or quantization. To show the relationships we can illustrate them in the following diagram similar to that given by I. Frenkel

Received by the editors September 9, 2009 and, in revised form, December 15, 2009.

2000 Mathematics Subject Classification. Primary 17B20.

Key words and phrases. Two-parameter quantum affine algebra, finite groups, wreath products, McKay correspondence.

The second author was the corresponding author for this paper.

(C)2011 American Mathematical Society Reverts to public domain 28 years from publication 
earlier. Our new algebra is shown here at the lowest vertex representing a new direction of generalization.

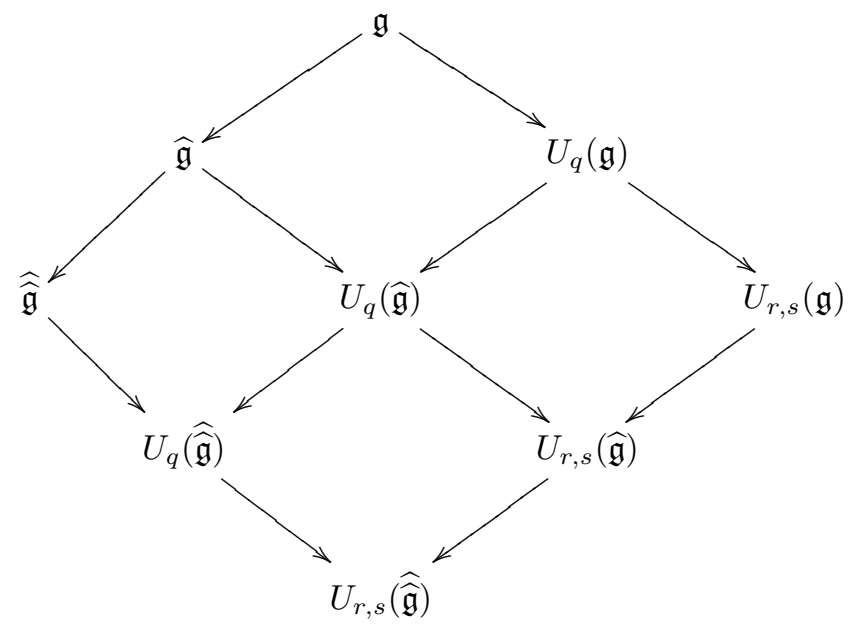

It is amazing that each vertex in the diagram admits a realization through McKay correspondence, and each level of complexity is achieved by replacing the finite group $\Gamma$ by $\Gamma \times \mathbb{C}^{\times}$and by $\Gamma \times \mathbb{C}^{\times} \times \mathbb{C}^{\times}$. The quantum parameter $q$ and $(r, s)$ are respectively represented by special characters on the groups $\mathbb{C}^{\times}$and $\mathbb{C}^{\times} \times \mathbb{C}^{\times}$.

At the early stage of the development of quantum groups it was already realized that there exist multi-parameter quantum groups $[\underline{R}, \mathbb{T}]$. Though two-parameter cases play an important role in the dual quantum picture, the development of twoparameter quantum groups only took a turn after more structures were revealed in a series of papers by Benkart and Witherspoon BW1, BW2, BW3 for type $A$. Shortly after, these generalizations to other types were given by Bergeron, Gao and $\mathrm{Hu}$ [BGH1, BGH2, BH]. Recently $\mathrm{Hu}$, Rosso and one of us found the two-parameter quantum affine algebras of ADE types [HRZ, Z] via their vertex representations [HZ] based on generalized Drinfeld realizations (cf. [J2]), which are the two-parameter analog of the Frenkel-Jing representations FJ. In all these works one realizes that the two-parameter analog, like its one-parameter case, amounts to a clever deformation of the natural number $n$ to the two-parameter quantum number:

$$
[n]=\frac{r^{n}-s^{n}}{r-s}=r^{n-1}+r^{n-2} s+\cdots+r s^{n-2}+s^{n-1} .
$$

Clearly when $r s=1$, the two-parameter quantum number degenerates to the oneparameter quantum number.

As in the previous construction of McKay correspondence and quantum toroidal algebras, we recover the basic representation of $U_{r, s}(\widehat{\mathfrak{g}})$ by choosing

$$
\xi=\gamma_{0} \otimes\left(\left(r s^{-1}\right)^{\frac{1}{2}}+\left(r^{-1} s\right)^{\frac{1}{2}}\right)-\pi \otimes 1_{\mathbb{C}^{\times} \times \mathbb{C}^{\times},}
$$

where $\gamma_{0}, 1_{\mathbb{C}^{\times} \times \mathbb{C}^{\times}}$are the trivial characters of $\Gamma$ and $\mathbb{C}^{\times} \times \mathbb{C}^{\times}$, respectively, $r$ and $s$ are two independent natural characters of $\mathbb{C}^{\times}$, and $\pi$ is the natural character of the imbedding of $\Gamma$ in $S L_{2}(\mathbb{C})$. The natural appearance of the two-parameter quantum toroidal algebra in this picture intrinsically shows its importance in the representation theory of two-parameter quantum affine algebras. 
In the first realization of the affine and toroidal Lie algebras via McKay correspondence [FJW1] the important role of wreath products was pointed out in Wang's earlier geometric work on Hilbert schemes $[\mathrm{W}$. It remains an interesting question whether there is a geometric connection of the new two-parameter quantum groups to Nakajima's quiver varieties $\mathbb{N}$.

The paper is organized as follows. Section 2 recalls the basic material of wreath products $\Gamma_{n}$ of symmetric groups associated with any finite group and the Hopf algebra structures in the representation ring. Section 3 studies the representation ring $R\left(\Gamma_{n} \times \mathbb{C}^{\times} \times \mathbb{C}^{\times}\right)$and weighted bilinear forms. Section 4 gives the twoparameter McKay weights for each finite subgroup of $S L_{2}(\mathbb{C})$. Section 5 defines the two-parameter Heisenberg algebra and realizes its canonical representation using group-theoretic data out of $\Gamma$. Section 6 gives the Frobenius-type characteristic map between $R_{\Gamma \times \mathbb{C}^{\times} \times \mathbb{C}^{\times}}$and $S_{\Gamma \times \mathbb{C}^{\times} \times \mathbb{C}^{\times}}$. Section 7 realizes the two-parameter quantum vertex operators using irreducible characters of $\Gamma_{n} \times \mathbb{C}^{\times} \times \mathbb{C}^{\times}$, and finally in Section 8 we introduce two-parameter quantum toroidal algebras and provide their realization via McKay correspondence, and in particular this also provides a group-theoretic realization of the basic representation of the two-parameter quantum affine algebras.

\section{WREATH PRODUCTS AND VERTEX REPRESENTATIONS}

2.1. The wreath product $\Gamma_{n}$. Let $\Gamma$ be a finite group and $n$ a nonnegative integer. The wreath product $\Gamma_{n}$ is the semidirect product of the $n$-th direct product $\Gamma^{n}=$ $\Gamma \times \cdots \times \Gamma$ and the symmetric group $S_{n}$ :

$$
\Gamma_{n}=\left\{(g, \sigma) \mid g=\left(g_{1}, \ldots, g_{n}\right) \in \Gamma^{n}, \sigma \in S_{n}\right\}
$$

with the group multiplication

$$
(g, \sigma) \cdot(h, \tau)=(g \sigma(h), \sigma \tau)
$$

where $S_{n}$ acts on $\Gamma^{n}$ by permuting the factors.

Let $\Gamma_{*}$ be the set of conjugacy classes of $\Gamma$ consisting of $c^{0}=\{1\}, c^{1}, \ldots, c^{\ell}$ and let $\Gamma^{*}$ be the set of $\ell+1$ irreducible characters: $\gamma_{0}, \gamma_{1}, \ldots, \gamma_{\ell}$, where $\gamma_{0}$ is the trivial character of $\Gamma$. The order of the centralizer of an element in the conjugacy class $c$ is denoted by $\zeta_{c}$, so the order of the conjugacy class $c$ is $|c|=|\Gamma| / \zeta_{c}$, where $|\Gamma|$ is the order of $\Gamma$.

A partition $\lambda=\left(\lambda_{1}, \lambda_{2}, \ldots, \lambda_{\ell}\right)$ is a decomposition of $n=|\lambda|=\lambda_{1}+\cdots+\lambda_{l}$ with nonnegative integers: $\lambda_{1} \geq \cdots \geq \lambda_{\ell} \geq 1$, where $\ell=\ell(\lambda)$ is called the length of the partition $\lambda$ and $\lambda_{i}$ are called the parts of $\lambda$. Another notation for $\lambda$ is

$$
\lambda=\left(1^{m_{1}} 2^{m_{2}} \cdots\right)
$$

with $m_{i}$ being the multiplicity of parts equal to $i$ in $\lambda$. Denote by $\mathcal{P}$ the set of all partitions of integers and by $\mathcal{P}(S)$ the set of all partition-valued functions on a set $S$. The weight of a partition-valued function $\rho=(\rho(s))_{s \in S}$ is defined to be $\|\rho\|=\sum_{s \in S}|\rho(s)|$. We also denote by $\mathcal{P}_{n}$ (resp. $\left.\mathcal{P}_{n}(S)\right)$ the subset of $\mathcal{P}$ (resp. $\mathcal{P}(S)$ ) of partitions with weight $n$.

It is well known that the conjugacy classes of $\Gamma_{n}$ are parameterized by partitionvalued functions on $\Gamma_{*}$. Let $x=(g, \sigma) \in \Gamma_{n}$, where $g=\left(g_{1}, \ldots, g_{n}\right) \in \Gamma^{n}$ and $\sigma \in S_{n}$ is presented as a product of disjoint cycles. For each cycle $\left(i_{1} i_{2} \cdots i_{k}\right)$ of $\sigma$, we define the cycle-product element $g_{i_{k}} g_{i_{k-1}} \cdots g_{i_{1}} \in \Gamma$, which is determined up to conjugacy in $\Gamma$ by $g$ and the cycle. For any conjugacy class $c \in \Gamma$ and each integer $i \geq 1$, the number of $i$-cycles in $\sigma$ whose cycle-product lies in $c$ will 
be denoted by $m_{i}(c)$. This gives rise to a partition $\rho(c)=\left(1^{m_{1}(c)} 2^{m_{2}(c)} \ldots\right)$ for $c \in \Gamma_{*}$. Thus we obtain a partition-valued function $\rho=(\rho(c))_{c \in \Gamma_{*}} \in \mathcal{P}\left(\Gamma_{*}\right)$ such that $\|\rho\|=\sum_{i, c} i m_{i}(\rho(c))=n$. This is called the type of the element $(g, \sigma)$. It is known $\mathrm{M}$ that two elements in the same conjugacy class have the same type and that there exists a one-to-one correspondence between the sets $\left(\Gamma_{n}\right)_{*}$ and $\mathcal{P}_{n}\left(\Gamma_{*}\right)$. We will say that $\rho$ is the type of the conjugacy class of $\Gamma_{n}$.

Given a class $c$ we denote by $c^{-1}$ the class $\left\{x^{-1} \mid x \in c\right\}$. For each $\rho \in \mathcal{P}\left(\Gamma_{*}\right)$ we also associate the partition-valued function

$$
\bar{\rho}=\left(\rho\left(c^{-1}\right)\right)_{c \in \Gamma_{*}} .
$$

Given a partition $\lambda=\left(1^{m_{1}} 2^{m_{2}} \ldots\right)$, we denote by $z_{\lambda}=\prod_{i \geq 1} i^{m_{i}} m_{i}$ ! the order of the centralizer of an element of cycle type $\lambda$ in $S_{|\lambda|}$. The order of the centralizer of an element $x=(g, \sigma) \in \Gamma_{n}$ of type $\rho=(\rho(c))_{c \in \Gamma_{*}}$ is given by

$$
Z_{\rho}=\prod_{c \in \Gamma_{*}} z_{\rho(c)} \zeta_{c}^{l(\rho(c))}
$$

2.2. Grothendieck ring $R_{\Gamma \times C \times \times C \times}$. Let $R_{\mathbb{Z}}(\Gamma)$ be the $\mathbb{Z}$-lattice generated by $\gamma_{i}$, $i=0, \ldots, r$, and let $R(\Gamma)=\mathbb{C} \otimes R_{\mathbb{Z}}(\Gamma)$ be the space of complex class functions on the group $\Gamma$. In the previous work on the McKay correspondence and vertex representations [W], FJW1], the Grothendieck ring $R_{\Gamma}=\bigoplus_{n \geq 0} R\left(\Gamma_{n}\right)$ was studied. In the quantum case, the Grothendieck ring was $R_{\Gamma \times \mathbb{C}^{\times}} \stackrel{=}{=} \bigoplus_{n \geq 0} R\left(\Gamma_{n} \times \mathbb{C}^{\times}\right)$ [FJW2]. In our two-parameter quantum case, we need to add another ring $R\left(\mathbb{C}^{\times}\right)$, the space of characters of $\mathbb{C}^{\times} \times \mathbb{C}^{\times}=\left\{\left(t_{1}, t_{2}\right) \in \mathbb{C} \times \mathbb{C} \mid t_{1}, t_{2} \neq 0\right\}$.

Let $r, s$ be the irreducible characters of $\mathbb{C}^{\times}$that send $t_{1}$ and $t_{2}$ to itself respectively. Then $R\left(\mathbb{C}^{\times} \times \mathbb{C}^{\times}\right)$is spanned by irreducible multiplicative characters $r^{m} s^{n}$, $m, n \in \mathbb{Z}$, where

$$
r^{m}\left(t_{1}\right)=t_{1}^{m}, \quad s^{n}\left(t_{2}\right)=t_{2}^{n} \quad t_{1}, t_{2} \in \mathbb{C}^{\times} .
$$

Thus $R\left(\mathbb{C}^{\times} \times \mathbb{C}^{\times}\right)$is identified with the ring $\mathbb{C}\left[r^{ \pm 1}, s^{ \pm 1}\right]$, and we have

$$
R\left(\Gamma \times \mathbb{C}^{\times} \times \mathbb{C}^{\times}\right)=R(\Gamma) \otimes R\left(\mathbb{C}^{\times} \times \mathbb{C}^{\times}\right)
$$

An element of $R\left(\Gamma \times \mathbb{C}^{\times} \times \mathbb{C}^{\times}\right)$can be written as a finite sum:

$$
f=\sum_{i} f_{i} \otimes r^{m_{i}} s^{n_{i}}, \quad f_{i} \in R(\Gamma), m_{i}, n_{i} \in \mathbb{Z}
$$

We can also view $f$ as a function on $\Gamma$ with values in the ring of Laurent polynomials $\mathbb{C}\left[r^{ \pm 1}, s^{ \pm 1}\right]$. In this case we will write $f^{r, s}$ to indicate the formal variables $r, s$; then $f^{r, s}(c)=\sum_{i} f_{i}(c) r^{m_{i}} s^{n_{i}} \in \mathbb{C}\left[r^{ \pm 1}, s^{ \pm 1}\right]$. As a function on $\Gamma \times \mathbb{C}^{\times} \times \mathbb{C}^{\times}$, we have $f\left(c, t_{1}, t_{2}\right)=\sum_{i} f_{i}(c) t_{1}^{m_{i}} t_{2}^{n_{i}}$.

Denote by $R_{\Gamma \times C^{\times} \times C^{\times}}$the following direct sum:

$$
R_{\Gamma \times C^{\times} \times C^{\times}}=\bigoplus_{n \geq 0} R\left(\Gamma_{n} \times \mathbb{C}^{\times} \times \mathbb{C}^{\times}\right) \simeq R_{\Gamma} \otimes \mathbb{C}\left[r^{ \pm 1}, s^{ \pm 1}\right] .
$$

2.3. Hopf algebra structure on $R_{\Gamma \times C^{\times} \times C^{\times}}$. The multiplication $m$ in $\mathbb{C}^{\times} \times \mathbb{C}^{\times}$ and the diagonal map $\mathbb{C}^{\times} \times \mathbb{C}^{\times} \stackrel{d}{\longrightarrow} \mathbb{C}^{\times} \times \mathbb{C}^{\times} \times \mathbb{C}^{\times} \times \mathbb{C}^{\times}$induce the Hopf algebra 
structure on $R\left(\mathbb{C}^{\times} \times \mathbb{C}^{\times}\right)$:

$$
\begin{aligned}
& m_{\mathbb{C}^{\times} \times C^{\times}}: R\left(\mathbb{C}^{\times} \times \mathbb{C}^{\times}\right) \otimes R\left(\mathbb{C}^{\times} \times \mathbb{C}^{\times}\right) \stackrel{\cong}{\longrightarrow} R\left(\mathbb{C}^{\times} \times \mathbb{C}^{\times} \times \mathbb{C}^{\times} \times \mathbb{C}^{\times}\right) \stackrel{d^{*}}{\longrightarrow} R\left(\mathbb{C}^{\times} \times \mathbb{C}^{\times}\right), \\
& (2.2) \quad \Delta_{\mathbb{C}^{\times} \times C^{\times}}: R\left(\mathbb{C}^{\times}\right) \stackrel{m^{*}}{\longrightarrow} R\left(\mathbb{C}^{\times} \times \mathbb{C}^{\times}\right) \stackrel{\cong}{\longrightarrow} R\left(\mathbb{C}^{\times}\right) \otimes R\left(\mathbb{C}^{\times}\right) .
\end{aligned}
$$

In terms of the basis $\left\{r^{m} s^{n}\right\}$ we have

$$
\begin{aligned}
r^{m_{1}} s^{n_{1}} \cdot r^{m_{2}} s^{n_{2}} & =r^{m_{1}+m_{2}} s^{n_{1}+n_{2}}, \\
\Delta\left(r^{m} s^{n}\right) & =r^{m} s^{n} \otimes r^{m} s^{n},
\end{aligned}
$$

where we abbreviate $\Delta_{\mathbb{C}^{\times} \times \mathbb{C}^{\times}}$by $\Delta$ and follow the convention of writing $a \cdot b=$ $m_{\mathbb{C}^{\times} \times \mathbb{C}^{\times}}(a \otimes b)$.

The antipode $S_{\mathbb{C} \times \times \mathbb{C} \times}$ and the counit $\epsilon_{\mathbb{C} \times \times \mathbb{C} \times}$ are given by

$$
S_{\mathbb{C}^{\times} \times \mathbb{C}^{\times}}\left(r^{m} s^{n}\right)=r^{-m} s^{-n}, \quad \epsilon_{\mathbb{C}^{\times} \times \mathbb{C}^{\times}}\left(r^{m} s^{n}\right)=\delta_{m-n, 0} .
$$

We extend the Hopf algebra structures on $R\left(\mathbb{C}^{\times} \times \mathbb{C}^{\times}\right)$and $R_{\Gamma}$ into a Hopf algebra structure on $R_{\Gamma \times \mathbb{C}^{\times} \times \mathbb{C}^{\times}}$using a standard procedure in Hopf algebras [A]. The multiplication and comultiplication are given by the respective composition of the following maps:

$$
\begin{aligned}
& m: R\left(\Gamma_{n} \times \mathbb{C}^{\times} \times \mathbb{C}^{\times}\right) \otimes R\left(\Gamma_{m} \times \mathbb{C}^{\times} \times \mathbb{C}^{\times}\right) \stackrel{\cong}{\longrightarrow} R\left(\Gamma_{n} \times \mathbb{C}^{\times} \times \mathbb{C}^{\times} \times \Gamma_{m} \times \mathbb{C}^{\times} \times \mathbb{C}^{\times}\right) \\
& \stackrel{1 \otimes m_{\mathbb{C}^{\times} \times \mathbb{C}^{\times}}}{\longrightarrow} R\left(\Gamma_{n} \times \Gamma_{m} \times \mathbb{C}^{\times} \times \mathbb{C}^{\times}\right) \stackrel{\operatorname{Ind} \otimes 1}{\longrightarrow} R\left(\Gamma_{n+m} \times \mathbb{C}^{\times} \times \mathbb{C}^{\times}\right) ; \\
& \Delta: R\left(\Gamma_{n} \times \mathbb{C}^{\times} \times \mathbb{C}^{\times}\right) \stackrel{\operatorname{Res} \otimes 1}{\longrightarrow} \bigoplus_{m=0}^{n} R\left(\Gamma_{n-m} \times \Gamma_{m} \times \mathbb{C}^{\times} \times \mathbb{C}^{\times}\right) \\
& \stackrel{1 \otimes \Delta_{\mathbb{C}^{\times} \times \mathbb{C} \times}}{\longrightarrow} \bigoplus_{m=0}^{n} R\left(\Gamma_{n-m} \times \Gamma_{m} \times \mathbb{C}^{\times} \times \mathbb{C}^{\times} \times \mathbb{C}^{\times} \times \mathbb{C}^{\times}\right) \\
& \stackrel{n}{\longrightarrow} \bigoplus_{m=0}^{n} R\left(\Gamma_{n-m} \times \mathbb{C}^{\times} \times \mathbb{C}^{\times}\right) \otimes R\left(\Gamma_{m} \times \mathbb{C}^{\times} \times \mathbb{C}^{\times}\right),
\end{aligned}
$$

where we have used the identification of $R\left(\mathbb{C}^{\times} \times \mathbb{C}^{\times}\right)$with $R\left(\mathbb{C}^{\times}\right) \otimes R\left(\mathbb{C}^{\times}\right)$in (2.1)(2.2). Also Ind $: R\left(\Gamma_{n} \times \Gamma_{m}\right) \longrightarrow R\left(\Gamma_{n+m}\right)$ denotes the induction functor and Res $: R\left(\Gamma_{n}\right) \longrightarrow R\left(\Gamma_{n-m} \times \Gamma_{m}\right)$ denotes the restriction functor.

The antipode is given by

$$
S\left(f\left(g,\left(t_{1}, t_{2}\right)\right)\right)=f\left(g^{-1},\left(t_{1}^{-1}, t_{2}^{-1}\right)\right), \quad g \in \Gamma, t_{1}, t_{2} \in \mathbb{C}^{\times} .
$$

In particular, $S(\gamma)(c)=\gamma\left(c^{-1}\right)$ for $\gamma \in \Gamma^{*}$. As we mentioned earlier, we may write $f \in R_{\Gamma \times \mathbb{C} \times \times \mathbb{C} \times}$ as

$$
f^{r, s}(g)=\sum_{i} f_{i}(g) r^{m_{i}} s^{n_{i}} .
$$

Then $S\left(f^{r, s}\right)(g)=\sum_{i} f_{i}\left(g^{-1}\right) r^{-m_{i}} s^{-n_{i}}$.

The counit $\epsilon$ is defined by

$$
\epsilon\left(R\left(\Gamma_{n} \times \mathbb{C}^{\times} \times \mathbb{C}^{\times}\right)\right)=0, \quad \text { if } n \neq 0,
$$

and $\epsilon$ on $R\left(\mathbb{C}^{\times} \times \mathbb{C}^{\times}\right)$is the counit of the Hopf algebra $R\left(\mathbb{C}^{\times} \times \mathbb{C}^{\times}\right)$. 


\section{A Weighted BILINEAR FORM ON $R\left(\Gamma_{n} \times \mathbb{C}^{\times} \times \mathbb{C}^{\times}\right)$}

3.1. A standard bilinear form on $R_{\Gamma \times \mathbb{C}^{\times} \times \mathbb{C}^{\times}}$. Let $f, g \in R\left(\Gamma \times \mathbb{C}^{\times} \times \mathbb{C}^{\times}\right)$ with $f=\sum_{i} f_{i} \otimes r^{m_{i}} s^{n_{i}}$ and $g=\sum_{i} g_{i} \otimes r^{k_{i}} s^{l_{i}}$. The $\mathbb{C}\left[r^{ \pm 1}, s^{ \pm 1}\right]$-valued standard $\mathbb{C}$-bilinear form on $R\left(\Gamma \times \mathbb{C}^{\times} \times \mathbb{C}^{\times}\right)$is defined as

$$
\begin{aligned}
\langle f, g\rangle_{\Gamma}^{r, s} & =\sum_{i, j}\left\langle f_{i}, g_{j}\right\rangle_{\Gamma} r^{m_{i}-k_{j}} s^{n_{i}-l_{j}} \\
& =\sum_{i, j} \sum_{c \in \Gamma_{*}} \zeta_{c}^{-1} f_{i}(c) g_{j}\left(c^{-1}\right) r^{m_{i}-k_{j}} s^{n_{i}-l_{j}},
\end{aligned}
$$

where we recall that $c^{-1}$ denotes the conjugacy class $\left\{x^{-1} \mid x \in c\right\}$ of $\Gamma$, and $\zeta_{c}$ is the order of the centralizer of the class $c$ in $\Gamma$. Sometimes we will also view the bilinear form as a function of $\left(t_{1}, t_{2}\right) \in \mathbb{C}^{\times} \times \mathbb{C}^{\times}$:

$$
\langle f, g\rangle_{\Gamma}^{r, s}\left(t_{1}, t_{2}\right)=\sum_{c \in \Gamma_{*}} \zeta_{c}^{-1} f\left(c,\left(t_{1}, t_{2}\right)\right) S\left(g\left(c,\left(t_{1}, t_{2}\right)\right)\right)
$$

The following is a direct consequence of the orthogonality of irreducible characters of $\Gamma$ :

$$
\begin{aligned}
\left\langle\gamma_{i} \otimes r^{m} s^{n}, \gamma_{j} \otimes r^{k} s^{l}\right\rangle_{\Gamma}^{r, s} & =\delta_{i j} r^{m-k} s^{n-l}, \\
\sum_{\gamma \in \Gamma^{*}} \gamma\left(c^{\prime}\right) S(\gamma)(c) & =\delta_{c, c^{\prime}} \zeta_{c}, \quad c, c^{\prime} \in \Gamma_{*} .
\end{aligned}
$$

Let $\langle, \quad\rangle_{\Gamma_{n}}^{r, s}$ be the $\mathbb{C}\left[r^{ \pm 1}, s^{ \pm 1}\right]$-valued bilinear form on $R\left(\Gamma_{n} \times \mathbb{C}^{\times} \times \mathbb{C}^{\times}\right)$. The $\mathbb{C}\left[r^{ \pm 1}, s^{ \pm 1}\right]$-valued standard bilinear form in $R_{\Gamma \times \mathbb{C}^{\times} \times \mathbb{C}^{\times}}$is defined in terms of the bilinear form on $R\left(\Gamma_{n} \times \mathbb{C}^{\times} \times \mathbb{C}^{\times}\right)$as follows:

$$
\langle u, v\rangle^{r, s}=\sum_{n \geq 0}\left\langle u_{n}, v_{n}\right\rangle_{\Gamma_{n}}^{r, s}
$$

where $u=\sum_{n} u_{n}$ and $v=\sum_{n} v_{n}$ with $u_{n}, v_{n} \in R\left(\Gamma_{n} \times \mathbb{C}^{\times} \times \mathbb{C}^{\times}\right)$.

3.2. A weighted bilinear form on $R_{\Gamma \times \mathbb{C}^{\times} \times \mathbb{C}^{\times}}$. A class function $\xi \in R_{\Gamma \times \mathbb{C}^{\times} \times \mathbb{C}^{\times}}$ is called self-dual if for all $x \in \Gamma,\left(t_{1}, t_{2}\right) \in \mathbb{C}^{\times} \times \mathbb{C}^{\times}$,

$$
\xi\left(x,\left(t_{1}, t_{2}\right)\right)=S\left(\xi\left(x,\left(t_{1}, t_{2}\right)\right)\right),
$$

or equivalently $\xi^{r, s}(x)=\xi^{r^{-1}, s^{-1}}\left(x^{-1}\right)$.

We fix a self-dual class function $\xi$. The tensor product of two representations $\gamma$ and $\beta$ in $R\left(\Gamma \times \mathbb{C}^{\times} \times \mathbb{C}^{\times}\right)$will be denoted by $\gamma * \beta$.

Let $a_{i j} \in \mathbb{C}\left[r^{ \pm 1}, s^{ \pm 1}\right]$ be the (virtual) multiplicity of $\gamma_{j}$ in $\xi * \gamma_{i}$, i.e.,

$$
\xi * \gamma_{i}=\sum_{j=0}^{r} a_{i j} \gamma_{j} .
$$

We denote by $A^{r, s}$ the $n \times n$ matrix $\left(a_{i j}\right)_{0 \leq i, j \leq n-1}$.

Associated to $\xi$ we introduce the following weighted bilinear form:

$$
\langle f, g\rangle_{\xi}^{r, s}=\langle\xi * f, g\rangle_{\Gamma}^{r, s}, \quad f, g \in R\left(\Gamma \times \mathbb{C}^{\times} \times \mathbb{C}^{\times}\right),
$$

where we use the superscript $r, s$ to indicate the $r, s$-dependence. The superscript $r, s$ is often omitted if the $r, s$-variable in characters $f$ and $g$ is clear from the 
context. The explicit formula of the bilinear form is given as follows:

$$
\begin{aligned}
\langle f, g\rangle_{\xi}^{r, s} & =\frac{1}{|\Gamma|} \sum_{x \in \Gamma} \xi^{r, s}(x) f^{r, s}(x) g^{r^{-1}, s^{-1}}\left(x^{-1}\right) \\
& =\sum_{c \in \Gamma_{*}} \zeta_{c}^{-1} \xi^{r, s}(c) f^{r, s}(c) g^{r^{-1}, s^{-1}}\left(c^{-1}\right)
\end{aligned}
$$

which is the average of the character $\xi * f * \bar{g}$ over $\Gamma$.

The self-duality of $\xi$ together with (3.3) implies that

$$
a_{i j}=\overline{a_{j i}}
$$

i.e., $A^{r, s}$ is a Hermitian-like matrix with the bar action given by $\bar{r}=s, \bar{s}=r$.

The orthogonality (3.1) implies that

$$
a_{i j}=\left\langle\gamma_{i}, \gamma_{j}\right\rangle_{\xi}^{r, s}
$$

Remark 3.1. If $\xi$ is the trivial character $\gamma_{0}$, then the weighted bilinear form becomes the standard one on $R\left(\Gamma \times \mathbb{C}^{\times} \times \mathbb{C}^{\times}\right)$.

3.3. A weighted bilinear form on $R\left(\Gamma_{n} \times \mathbb{C}^{\times} \times \mathbb{C}^{\times}\right)$. Let $V$ be a $\Gamma \times \mathbb{C}^{\times} \times \mathbb{C}^{\times}$module which affords a character $\gamma$ in $R\left(\Gamma_{n} \times \mathbb{C}^{\times} \times \mathbb{C}^{\times}\right)$. We can decompose $V$ as follows:

$$
V=\bigoplus_{i} V_{i} \otimes \mathbb{C}\left(k_{i}, l_{i}\right)
$$

where $V_{i}$ is a (virtual) $\Gamma$-module in $R(\Gamma)$ and $\mathbb{C}\left(k_{i}, l_{i}\right)$ is the one-dimensional $\mathbb{C}^{\times} \times \mathbb{C}^{\times}$-module afforded by the character $r^{k_{i}} s^{l_{i}}$.

The $n$-th outer tensor product $V^{\otimes n}$ of $V$ can be regarded naturally as a representation of the wreath product $\left(\Gamma \times \mathbb{C}^{\times} \times \mathbb{C}^{\times}\right)_{n}$ via a permutation of the factors and the usual direct product action. More precisely, note that $\Gamma \times \mathbb{C}^{\times} \times \mathbb{C}^{\times}$can be viewed as a subgroup of $\left(\Gamma \times \mathbb{C}^{\times} \times \mathbb{C}^{\times}\right)_{n}$ by the diagonal inclusion from $\mathbb{C}^{\times} \times \mathbb{C}^{\times}$to $\left(\mathbb{C}^{\times} \times \mathbb{C}^{\times}\right)^{n}:$

$$
\Gamma_{n} \times \mathbb{C}^{\times} \times \mathbb{C}^{\times} \longrightarrow\left(\Gamma^{n} \times \mathbb{C}^{\times} \times \mathbb{C}^{\times n}\right) \rtimes S_{n}=\left(\Gamma \times \mathbb{C}^{\times} \times \mathbb{C}^{\times}\right)_{n} .
$$

This provides a natural $\Gamma_{n} \times \mathbb{C}^{\times} \times \mathbb{C}^{\times}$-module structure on $V^{\otimes n}$. We denote its character by $\eta_{n}(\gamma)$. Explicitly we have

$$
\left(g, \sigma,\left(t_{1}, t_{2}\right)\right) \cdot\left(v_{1} \otimes \cdots \otimes v_{n}\right)=\left(g_{1},\left(t_{1}, t_{2}\right)\right) v_{\sigma^{-1}(1)} \otimes \cdots \otimes\left(g_{n},\left(t_{1}, t_{2}\right)\right) v_{\sigma^{-1}(n)},
$$

where $g=\left(g_{1}, \ldots, g_{n}\right) \in \Gamma^{n}$.

Let $\varepsilon_{n}$ be the (1-dimensional) sign representation of $\Gamma_{n}$ so that $\Gamma^{n}$ acts trivially while letting $S_{n}$ act as a sign representation. We denote by $\varepsilon_{n}(\gamma) \in R\left(\Gamma_{n} \times \mathbb{C}^{\times} \times \mathbb{C}^{\times}\right)$ the character of the tensor product of $\varepsilon_{n} \otimes 1$ and $V^{\otimes n}$.

The weighted bilinear form on $R\left(\Gamma_{n} \times \mathbb{C}^{\times} \times \mathbb{C}^{\times}\right)$is now defined by

$$
\langle f, g\rangle_{\xi, \Gamma_{n}}^{r, s}=\left\langle\eta_{n}(\xi) * f, g\right\rangle_{\Gamma_{n}}^{r, s}, \quad f, g \in R\left(\Gamma_{n} \times \mathbb{C}^{\times} \times \mathbb{C}^{\times}\right) .
$$

We shall see in Corollary 6.4 that $\eta_{n}(\xi)$ is self-dual if the class function $\xi$ is invariant under the antipode $S$. In such a case the matrix of the bilinear form $\langle,\rangle_{\xi}^{r, s}$ is equal to its adjoint (transpose and bar action). 
We can naturally extend $\eta_{n}$ to a map from $R(\Gamma) \otimes r^{m} s^{n}$ to $R\left(\Gamma_{n} \times \mathbb{C}^{\times} \times \mathbb{C}^{\times}\right)$as follows (cf. [W]). In particular, if $\beta$ and $\gamma$ are characters of the representations $V$ and $W$ of $\Gamma$, respectively, then

$$
\begin{aligned}
& =\sum_{m=0}^{n} \operatorname{Ind}_{\Gamma_{n-m} \times \mathbb{C}^{\times} \times \mathbb{C}^{\times} \times \Gamma_{m} \times \mathbb{C}^{\times} \times \mathbb{C}^{\times}}^{\Gamma_{n} \times \mathbb{C}^{\times} \times \mathbb{C}^{\times}}\left[\eta_{n-m}\left(\beta \otimes r^{m} s^{n}\right) \otimes \eta_{m}\left(\gamma \otimes r^{k} s^{l}\right)\right], \\
& =\sum_{m=0}^{n}(-1)^{m} \operatorname{Ind}_{\Gamma_{n-m} \times \mathbb{C}^{\times} \times \mathbb{C}^{\times} \times \Gamma_{m} \times \mathbb{C}^{\times} \times \mathbb{C}^{\times}}\left[\eta_{n-m}\left(\beta \otimes r^{m} s^{n}\right) \otimes \varepsilon_{m}\left(\gamma \otimes r^{k} s^{l}\right)\right] .
\end{aligned}
$$

On $R_{\Gamma \times \mathbb{C}^{\times} \times \mathbb{C}^{\times}}=\bigoplus_{n} R\left(\Gamma_{n} \times \mathbb{C}^{\times} \times \mathbb{C}^{\times}\right)$the weighted bilinear form is given by

$$
\langle u, v\rangle_{\xi}^{r, s}=\sum_{n \geq 0}\left\langle u_{n}, v_{n}\right\rangle_{\xi, \Gamma_{n}}^{r, s},
$$

where $u=\sum_{n} u_{n}$ and $v=\sum_{n} v_{n}$ with $u_{n}, v_{n} \in R\left(\Gamma_{n} \times \mathbb{C}^{\times} \times \mathbb{C}^{\times}\right)$.

The bilinear form $\langle,\rangle_{\xi}^{r, s}$ on $R_{\Gamma \times \mathbb{C} \times \times \mathbb{C} \times}$ is $\mathbb{C}$-bilinear and takes values in $\mathbb{C}\left[r^{ \pm 1}\right.$, $\left.s^{ \pm 1}\right]$. When $n=1$, it reduces to the weighted bilinear form defined on $R_{\Gamma \times \mathbb{C}^{\times} \times \mathbb{C}^{\times}}$.

We will often omit the superscript $r, s$ and use the notation $\langle,\rangle_{\xi}$ for the weighted bilinear form on $R_{\Gamma \times \mathbb{C}^{\times} \times \mathbb{C}^{\times}}$.

\section{Two-Parameter quantum McKay Weights}

4.1. Two-parameter quantum McKay correspondence. Let $d_{i}=\gamma_{i}\left(c^{0}\right)$ be the dimension of the irreducible representation of $\Gamma$ corresponding to the character $\gamma_{i}$

The following generalizes a result of McKay [Mc].

Proposition 4.1. For each class $c \in \Gamma_{*}$ the column vector

$$
v(c)=\left(\gamma_{0}(c), \gamma_{1}(c), \ldots, \gamma_{n-1}(c)\right)^{t}
$$

is an eigenvector of the $n \times n$-matrix $A^{r, s}=\left(\left\langle\gamma_{i}, \gamma_{j}\right\rangle_{\xi}^{r, s}\right)$ with eigenvalue $\xi^{r, s}(c)$. In particular $\left(d_{0}, d_{1}, \ldots, d_{n-1}\right)$ is an eigenvector of $A^{r, s}$ with eigenvalue $\xi^{r, s}\left(c^{0}\right)$.

Proof. We compute directly that

$$
\begin{aligned}
\sum_{k=0}^{r}\left\langle\gamma_{i}, \gamma_{k}\right\rangle_{\xi}^{r, s} \gamma_{k}(c) & =\sum_{k} \sum_{c^{\prime} \in \Gamma_{*}} \zeta_{c^{\prime}}^{-1} \xi^{r, s}\left(c^{\prime}\right) \gamma_{i}\left(c^{\prime}\right) \gamma_{k}\left(c^{-1}\right) \gamma_{k}(c) \\
& =\sum_{c^{\prime} \in \Gamma_{*}} \zeta_{c^{\prime}}^{-1} \xi^{r, s}\left(c^{\prime}\right) \gamma_{i}\left(c^{\prime}\right) \sum_{k} \gamma_{k}\left(c^{\prime-1}\right) \gamma_{k}(c) \\
& =\sum_{c^{\prime} \in \Gamma_{*}} \zeta_{c^{\prime}}^{-1} \xi^{r, s}\left(c^{\prime}\right) \gamma_{i}\left(c^{\prime}\right) \zeta_{c} \delta_{c c^{\prime}} \\
& =\xi^{r, s}(c) \gamma_{i}(c)
\end{aligned}
$$

Let $\pi$ be an irreducible faithful representation $\pi$ of $\Gamma$ of dimension $d$. For each integer $n$ we define the $r, s$-integer $[n]$ that can be viewed as a character of $\mathbb{C}^{\times} \times \mathbb{C}^{\times}$ 
by

$$
[n]=\frac{r^{n}-s^{n}}{r-s}=r^{n-1}+r^{n-2} s+\cdots+r s^{n-2}+s^{n-1} .
$$

We take the following special class function:

$$
\xi=\gamma_{0} \otimes[d](r s)^{-\frac{d}{4}}-\pi \otimes 1_{\mathbb{C}^{\times} \times \mathbb{C}^{\times}},
$$

where we have also used the symbol $\pi$ for the corresponding character, and $1_{\mathbb{C}^{\times} \times \mathbb{C}^{\times}}$ $=r^{0} s^{0}$ is the trivial character of $\mathbb{C}^{\times} \times \mathbb{C}^{\times}$.

Similar to the one-parameter quantum case, we have the following fact (cf. [FJW2]).

Proposition 4.2. The weighted bilinear form associated to (4.1) is nondegenerate. If $\pi$ is an embedding of $\Gamma$ into $S U_{d}$ and $t \neq 1$ is a nonnegative real number, then the weighted bilinear form evaluated on $t$ is positive definite.

Remark 4.3. The matrix $A^{1,1}$ is integral, and the entries of $A^{r, s}$ are the $r, s$ numbers of the corresponding entries in $A^{1,1}$ when $r \geq 2$.

4.2. Two quantum McKay weights. Letting $\Gamma$ be a finite subgroup of $S U_{2}$, we introduce the first distinguished self-dual class function

$$
\xi=\gamma_{0} \otimes\left(\left(r s^{-1}\right)^{\frac{1}{2}}+\left(r^{-1} s\right)^{\frac{1}{2}}\right)-\pi \otimes 1_{\mathbb{C}^{\times} \times \mathbb{C}^{\times}},
$$

where $\pi$ is the character of the embedding of $\Gamma$ in $S U_{2}$.

The matrix of the weighted bilinear form $\langle,\rangle_{\xi}$ (cf. (3.4) ) has the following entries:

$$
a_{i j}= \begin{cases}\left(r s^{-1}\right)^{\frac{1}{2}}+\left(r^{-1} s\right)^{\frac{1}{2}}, & \text { if } i=j, \\ -1, & \text { if }\left\langle\gamma_{i}, \gamma_{j}\right\rangle_{\xi}^{1}=-1 .\end{cases}
$$

In particular when $r=s=1$ the matrix $\left(a_{i j}^{1,1}\right)$ coincides with the extended Cartan matrix of ADE type according to the five classes of finite subgroups of $\mathrm{SU}_{2}$ : the cyclic, binary dihedral, tetrahedral, octahedral, and icosahedral groups. McKay [Mc gave a direct correspondence between a finite subgroup of $\mathrm{SU}_{2}$ and the affine Dynkin diagram $D$ of ADE type. Each irreducible character $\gamma_{i}$ corresponds to a vertex of $D$, and the number of edges between $\gamma_{i}$ and $\gamma_{j}(i \neq j)$ is equal to $\left|\left\langle\gamma_{i}, \gamma_{j}\right\rangle_{\xi}^{1,1}\right|$, where $\left\langle\gamma_{i}, \gamma_{j}\right\rangle_{\xi}^{1,1}=a_{i j}^{1}$ are the entries of matrix $A^{1,1}$ of the weighted bilinear form $\langle,\rangle_{\xi}^{1,1}$. For this reason we will call our matrix $A^{r, s}=\left(a_{i j}\right)=$ $\left(\left\langle\gamma_{i}, \gamma_{j}\right\rangle_{\xi}^{r, s}\right)$ the quantum Cartan matrix.

\section{Two-Parameter quantum Heisenberg algebras and $\Gamma_{n}$}

5.1. Two-parameter Heisenberg algebra $\widehat{\mathfrak{h}}_{\Gamma, \xi}$. Let $\widehat{\mathfrak{h}}_{\Gamma, \xi}$ be the infinite-dimensional Heisenberg algebra over $\mathbb{C}\left[r^{ \pm 1}, s^{ \pm 1}\right]$, associated with $\Gamma$ and $\xi \in R\left(\Gamma_{n} \times \mathbb{C}^{\times}\right.$ $\left.\times \mathbb{C}^{\times}\right)$, with generators $a_{m}(c), c \in \Gamma_{*}, m \in \mathbb{Z}$ and a central element $C$ subject to the following commutation relations:

$$
\left[a_{m}\left(c^{-1}\right), a_{n}\left(c^{\prime}\right)\right]=m \delta_{m,-n} \delta_{c, c^{\prime}} \zeta_{c} \xi_{r^{m}, s^{m}}(c) C, \quad c, c^{\prime} \in \Gamma_{*} .
$$

For $m \in \mathbb{Z}, \gamma \in \Gamma^{*}$ and $k, l \in \mathbb{Z}$ we define

$$
a_{m}\left(\gamma \otimes r^{k} s^{l}\right)=\sum_{c \in \Gamma_{*}} \zeta_{c}^{-1} \gamma(c) a_{m}(c) r^{m k} s^{m l}
$$


and then extend it to $R\left(\Gamma_{n} \times \mathbb{C}^{\times} \times \mathbb{C}^{\times}\right)$linearly over $\mathbb{C}$. Thus we have for $\gamma \in$ $R\left(\Gamma_{n} \times \mathbb{C}^{\times} \times \mathbb{C}^{\times}\right)$,

$$
a_{m}(\gamma)=\sum_{c \in \Gamma_{*}} \zeta_{c}^{-1} \gamma_{r^{m}, s^{m}}(c) a_{m}(c) .
$$

In particular we have $a_{m}\left(\gamma \otimes r^{k} s^{l}\right)=a_{m}(\gamma) r^{m k} s^{m l}$.

It follows immediately from the orthogonality (3.1) of the irreducible characters of $\Gamma$ that for each $c \in \Gamma_{*}$,

$$
a_{m}(c)=\sum_{\gamma \in \Gamma^{*}} S(\gamma(c)) a_{m}(\gamma) .
$$

Note that this formula is also valid if the summation runs through $\Gamma^{*} \otimes r^{k} s^{l}$ with fixed $k$ and $l$.

Proposition 5.1. The Heisenberg algebra $\widehat{\mathfrak{h}}_{\Gamma, \xi}$ has a new basis given by $a_{n}(\gamma)$ and $C\left(n \in \mathbb{Z}, \gamma \in \Gamma^{*}\right)$ over $\mathbb{C}\left[r^{ \pm 1}, s^{ \pm 1}\right]$ with the following relations:

$$
\left[a_{m}(\gamma), a_{n}\left(\gamma^{\prime}\right)\right]=m \delta_{m,-n}\left\langle\gamma, \gamma^{\prime}\right\rangle_{\xi}^{r^{m}, s^{m}} C .
$$

Proof. This is proved by a direct computation using equations (5.1): (3.3) and (3.1):

$$
\begin{aligned}
{\left[a_{m}(\gamma), a_{n}\left(\gamma^{\prime}\right)\right] } & =\sum_{c, c^{\prime} \in \Gamma_{*}} \zeta_{c}^{-1} \zeta_{c^{\prime}}^{-1} \gamma(c) \gamma^{\prime}\left(c^{\prime}\right)\left[a_{m}(c), a_{n}\left(c^{\prime}\right)\right] \\
& =m \delta_{m,-n} \sum_{c, c^{\prime} \in \Gamma_{*}} \zeta_{c}^{-1} \zeta_{c^{\prime}}^{-1} \gamma(c) \gamma^{\prime}\left(c^{\prime}\right) \delta_{c^{-1}, c^{\prime}} \zeta_{c} \xi_{r^{m}, s^{m}}(c) C \\
& =m \delta_{m,-n} \sum_{c \in \Gamma_{*}} \zeta_{c}^{-1} \gamma(c) \gamma^{\prime}\left(c^{-1}\right) \xi_{r^{m}, s^{m}}(c) C \\
& =m \delta_{m,-n}\left\langle\gamma, \gamma^{\prime}\right\rangle_{\xi}^{r^{m}, s^{m}} C .
\end{aligned}
$$

5.2. Action of $\widehat{\mathfrak{h}}_{\Gamma, \xi}$ on the space $S_{\Gamma \times \mathbb{C} \times \times \mathbb{C} \times}$. Let $S_{\Gamma \times \mathbb{C} \times \times \mathbb{C} \times}$ be the symmetric algebra generated by $a_{-n}(\gamma), n \in \mathbb{N}, \gamma \in \Gamma_{*}$ over $\mathbb{C}\left[r^{ \pm 1}, s^{ \pm 1}\right]$. We define $a_{-n}(\gamma \otimes$ $\left.r^{k} s^{l}\right)=a_{-n}(\gamma) r^{-k n} s^{-l n}$ and the natural degree operator on the space $S_{\Gamma \times \mathbb{C}^{\times} \times \mathbb{C}^{\times}}$ by

$$
\operatorname{deg}\left(a_{-n}\left(\gamma \otimes r^{k} s^{l}\right)\right)=n
$$

which makes $S_{\Gamma \times \mathbb{C}^{\times} \times \mathbb{C}^{\times}}$into a $\mathbb{Z}_{+}$-graded algebra.

The space $S_{\Gamma \times \mathbb{C}^{\times} \times \mathbb{C}^{\times}}$affords a natural realization of the Heisenberg algebra $\widehat{\mathfrak{h}}_{\Gamma, \xi}$ with $C=1$. Since $a_{-n}\left(\gamma \otimes r^{k} s^{l}\right)=r^{-n k} s^{-n l} a_{-n}(\gamma)$, it is enough to describe the action for $a_{-n}(\gamma)$. The central element $C$ acts as the identity operator. For $n>0$, $a_{-n}(\gamma)$ act as multiplication operators on $S_{\Gamma \times \mathbb{C}^{\times} \times \mathbb{C}^{\times}}$. The element $a_{n}(\gamma), n \geq 0$ acts as a differential operator through contraction:

$$
\begin{gathered}
a_{n}(\gamma) \cdot a_{-n_{1}}\left(\alpha_{1}\right) a_{-n_{2}}\left(\alpha_{2}\right) \ldots a_{-n_{k}}\left(\alpha_{k}\right) \\
=\sum_{i=1}^{k}\left\langle\gamma, \alpha_{i}\right\rangle_{\xi}^{r^{n}, s^{n}} a_{-n_{1}}\left(\alpha_{1}\right) a_{-n_{2}}\left(\alpha_{2}\right) \ldots \check{a}_{-n_{i}}\left(\alpha_{i}\right) \ldots a_{-n_{k}}\left(\alpha_{k}\right) .
\end{gathered}
$$

Here $n_{i}>0, \alpha_{i} \in R(\Gamma)$ for $i=1, \ldots, k$, and $\check{a}_{-n_{i}}\left(\alpha_{i}\right)$ means that the indicated term is deleted. In this case, $S_{\Gamma \times \mathbb{C}^{\times} \times \mathbb{C}^{\times}}$is an irreducible representation of $\widehat{\mathfrak{h}}_{\Gamma, \xi}$ with the unit 1 as the highest weight vector. 
5.3. The bilinear form on $S_{\Gamma \times \mathbb{C}^{\times} \times \mathbb{C}^{\times}}$. As a $\widehat{\mathfrak{h}}_{\Gamma, \xi^{-m o d u l e}}$ the space $S_{\Gamma \times \mathbb{C}^{\times} \times \mathbb{C}^{\times}}$ admits a bilinear form $\langle,\rangle_{\xi}^{\prime}$ over $\mathbb{C}\left[r^{ \pm 1}, s^{ \pm 1}\right]$ characterized by

$$
\begin{aligned}
\langle 1,1\rangle_{\xi}^{\prime} & =1, \\
\langle a u, v\rangle_{\xi}^{\prime} & =\left\langle u, a^{*} v\right\rangle_{\xi}^{\prime}, \quad a \in \widehat{\mathfrak{h}}_{\Gamma, \xi},
\end{aligned}
$$

with the adjoint map $*$ on $\widehat{\mathfrak{h}}_{\Gamma, \xi}$ given by

$$
a_{n}\left(\gamma \otimes r^{k} s^{l}\right)^{*}=a_{-n}\left(\gamma \otimes r^{k} s^{l}\right), \quad n \in \mathbb{Z} .
$$

Note that the adjoint map $*$ is a $\mathbb{C}$-linear anti-homomorphism of $\widehat{\mathfrak{h}}_{\Gamma, \xi}$, and $r^{*}=$ $\bar{r}, s^{*}=\bar{s}$. We still use the same symbol $*$ to denote the Hermitian-like dual, since it clearly generalizes the $*$-action on the deformed Cartan matrix.

For any partition $\lambda=\left(\lambda_{1}, \lambda_{2}, \ldots\right)$ and $\gamma \in \Gamma^{*}$, we define

$$
a_{-\lambda}(\gamma)=a_{-\lambda_{1}}(\gamma) a_{-\lambda_{2}}(\gamma) \ldots
$$

For $\rho=(\rho(\gamma))_{\gamma \in \Gamma^{*}} \in \mathcal{P}\left(\Gamma^{*}\right)$, we define

$$
a_{-\rho \otimes r^{k} s^{l}}=r^{-k\|\rho\|} s^{-l\|\rho\|} \prod_{\gamma \in \Gamma^{*}} a_{-\rho(\gamma)}(\gamma) .
$$

It is clear that for fixed $k, l \in \mathbb{Z}$ the elements $a_{-\rho \otimes r^{k} s^{l}}, \rho \in \mathcal{P}\left(\Gamma^{*}\right)$ form a basis of $S_{\Gamma \times \mathbb{C} \times \times \mathbb{C} \times}$ over $\mathbb{C}\left[r^{ \pm 1}, s^{ \pm 1}\right]$.

Given a partition $\lambda=\left(\lambda_{1}, \lambda_{2}, \ldots\right)$ and $c \in \Gamma_{*}$, we define

$$
a_{-\lambda}\left(c \otimes r^{k} s^{l}\right)=r^{-k|\lambda|} s^{-l|\lambda|} a_{-\lambda_{1}}(c) a_{-\lambda_{2}}(c) \ldots
$$

For any $\rho=(\rho(c))_{c \in \Gamma_{*}} \in \mathcal{P}\left(\Gamma_{*}\right)$ and $k \in \mathbb{Z}$, we define

$$
a_{-\rho \otimes r^{k} s^{l}}^{\prime}=r^{-k\|\rho\|} s^{-l\|\rho\|} \prod_{c \in \Gamma_{*}} a_{-\rho(c)}(c) .
$$

It follows from Proposition 5.1 that

$$
\left\langle a_{-\rho \otimes r^{m} s^{n}}^{\prime}, a_{-\bar{\sigma} \otimes r^{k} s^{l}}^{\prime}\right\rangle_{\xi}^{\prime}=\delta_{\rho, \sigma} r^{\|\rho\|(k-m)} s^{\|\rho\|(l-n)} Z_{\rho} \prod_{c \in \Gamma_{*}} \prod_{i \geq 1} \xi_{q^{i}}(c)^{m_{i}(\rho(c))},
$$

where $\rho, \sigma \in \mathcal{P}\left(\Gamma_{*}\right)$. Note that $S\left(a_{-\rho \otimes r^{k} s^{l}}^{\prime}\right)=a_{-\bar{\rho} \otimes r^{-k} s^{-l}}^{\prime}$, where we recall that $\bar{\rho} \in \mathcal{P}\left(\Gamma_{*}\right)$ is the partition-valued function given by $c \mapsto \rho\left(c^{-1}\right), c \in \Gamma$.

\section{THE CHARACTERISTIC MAP AS AN ISOMETRY}

6.1. The characteristic map ch. Let $\Psi: \Gamma_{n} \rightarrow S_{\Gamma \times \mathbb{C}^{\times} \times \mathbb{C}^{\times}}$be the map defined by $\Psi(x)=a_{-\rho}^{\prime}$ if $x \in \Gamma_{n}$ is of type $\rho$.

We define a $\mathbb{C}$-linear map $c h: R_{\Gamma \times \mathbb{C}^{\times} \times \mathbb{C}^{\times}} \longrightarrow S_{\Gamma \times \mathbb{C}^{\times} \times \mathbb{C}^{\times}}$by letting

$$
\begin{aligned}
\operatorname{ch}(f) & =\langle f, \Psi\rangle_{\Gamma_{n}} \\
& =\sum_{\rho \in \mathcal{P}\left(\Gamma_{*}\right)} Z_{\rho}^{-1} S(f(\rho)) a_{-\rho}^{\prime},
\end{aligned}
$$

where $f(\rho) \in \mathbb{C}\left[r^{ \pm 1}, s^{ \pm 1}\right]$ is the value of $f$ at the elements of type $\rho$. The map $c h$ is called the characteristic map. This generalizes the definition of the characteristic map in the classical setting (cf. [M, FJW1, FJW2]).

The space $S_{\Gamma \times \mathbb{C}^{\times} \times \mathbb{C}^{\times}}$can also be interpreted as follows. The element $a_{-n}(\gamma), n>$ $0, \gamma \in \Gamma^{*}$ is identified as the $n$-th power sum in a sequence of variables $y_{g}=\left(y_{i \gamma}\right)_{i \geq 1}$. By the commutativity among $a_{-n}(\gamma)\left(\gamma \in \Gamma^{*}, n>0\right)$ and dimension counting it 
is clear that the space $S_{\Gamma \times \mathbb{C}^{\times} \times \mathbb{C}^{\times}}$is isomorphic with the space $\Lambda_{\Gamma}$ of symmetric functions indexed by $\Gamma^{*}$ tensored with $\mathbb{C}\left[r^{ \pm 1}, s^{ \pm 1}\right]$ (cf. [M]).

Denote by $c_{n}\left(c \in \Gamma_{*}\right)$ the conjugacy class in $\Gamma_{n}$ of elements $(x, p) \in \Gamma_{n}$ such that $p$ is an $n$-cycle and $x \in c$. Denote by $\sigma_{n}\left(c \otimes r^{k} s^{l}\right)$ the class function on $\Gamma_{n} \times \mathbb{C}^{\times} \times \mathbb{C}^{\times}$ which takes values $n \zeta_{c} t_{1}^{-n k} t_{2}^{-n l}$ (i.e., the order of the centralizer of an element in the class $c_{n}$ times $t_{1}^{-n k} t_{2}^{-n l}$ ) on elements in the class $c_{n} \times t_{1} t_{2}$ and 0 elsewhere. For $\rho=\left\{m_{r}(c)\right\}_{r \geq 1, c \in \Gamma_{*}} \in \mathcal{P}_{n}\left(\Gamma^{*}\right)$ and $k \in \mathbb{Z}$

$$
\sigma_{\rho \otimes r^{k} s^{l}}=r^{n l} s^{n k} \prod_{a \geq 1, c \in \Gamma_{*}} \sigma_{a}(c)^{m_{a}(c)}
$$

is the class function on $\Gamma_{n} \times \mathbb{C}^{\times} \times \mathbb{C}^{\times}$which takes values $Z_{\rho} t_{1}^{-n k} t_{2}^{-n l}$ on the conjugacy class of type $\rho \times t_{1} t_{2}$ and 0 elsewhere. Given $\gamma \in \Gamma^{*}$ and $k, l \in \mathbb{Z}$, we denote by $\sigma_{n}\left(\gamma \otimes r^{k} s^{l}\right)$ the class function on $\Gamma_{n} \times \mathbb{C}^{\times} \times \mathbb{C}^{\times}$which takes values $n \gamma(c) t_{1}^{-n k} t_{2}^{-n l}$ on elements in the class $c_{n} \times t_{1} t_{2}\left(c \in \Gamma_{*}\right)$ and 0 elsewhere.

Lemma 6.1. The map ch sends $\sigma_{\rho \otimes r^{k} s^{l}}$ to $a_{-\rho \otimes r^{k} s^{l}}^{\prime}$. In particular, it sends $\sigma_{n}(\gamma \otimes$ $\left.r^{k} s^{l}\right)$ to $a_{-n}\left(\gamma \otimes r^{k} s^{l}\right)$ in $S_{\Gamma \times \mathbb{C}^{\times} \times \mathbb{C}^{\times}}$.

Proof. This is verified by the definition of $c h$ (6.1) and the character values of $\sigma_{n}$ defined above.

Proposition 6.2. Given $\gamma \in \Gamma^{*}$, the character value of $\eta_{n}\left(\gamma \otimes r^{k} s^{l}\right)$ on the conjugacy class $c_{\rho}$ of type $\rho=(\rho(c))_{c \in \Gamma_{*}}$ is given by

$$
\eta_{n}\left(\gamma \otimes r^{k} s^{l}\right)\left(c_{\rho}\right)=\prod_{c \in \Gamma_{*}} \gamma(c)^{l(\rho(c))} r^{n k} s^{n l} .
$$

In particular, we have $\eta_{n}\left(\gamma \otimes r^{k} s^{l}\right)=\eta_{n}(\gamma) r^{n k} s^{n l}$.

Proof. We first let $(g, \sigma)$ be an element of $\Gamma_{n}$ such that $\sigma$ is a cycle of length $n$, say $\sigma=(12 \cdots n)$. Let $\left\{e_{i}\right\}$ be a basis of $V$, and let $\gamma \otimes r^{k} s^{l}$ be afforded by the action: $(h, t) e_{j}=\sum_{i} c_{i j}(h) t^{k} e_{i}$, where $h \in \Gamma$. We then have

$$
\begin{aligned}
& (g, \sigma, t) .\left(e_{j_{1}} \otimes e_{j_{2}} \otimes \cdots \otimes e_{j_{n}}\right) \\
& =\left(g_{1}, t\right) e_{j_{n}} \otimes\left(g_{2}, t\right) e_{j_{1}} \otimes \cdots \otimes\left(g_{n}, t\right) e_{j_{n-1}} \\
& =\sum_{i_{1}, \ldots, i_{n}} t^{k n} c_{i_{n} j_{n}}\left(g_{1}\right) c_{i_{1} j_{1}}\left(g_{2}\right) \cdots c_{i_{n-1} j_{n-1}}\left(g_{n}\right) e_{i_{n}} \otimes e_{i_{1}} \cdots \otimes e_{i_{n-1}} .
\end{aligned}
$$

It follows that

$$
\begin{aligned}
\eta_{n}\left(\gamma \otimes r^{k} s^{l}\right)\left(c_{\rho}, t\right) & =\operatorname{trace}(g, \sigma, t) \\
& =\sum_{j_{1}, \ldots, j_{n}} t^{k n} c_{j_{1} j_{n}}\left(g_{1}\right) c_{j_{2} j_{1}}\left(g_{2}\right) \cdots c_{j_{n} j_{n-1}}\left(g_{n}\right) \\
& =\operatorname{trace} t^{k n} a\left(g_{n}\right) a\left(g_{n-1}\right) \ldots a\left(g_{1}\right) \\
& =\operatorname{trace} t^{k n} a\left(g_{n} g_{n-1} \ldots g_{1}\right)=\gamma(c) r^{k n} s^{l n}(t) .
\end{aligned}
$$

Given $x \times y \in \Gamma_{n}$ where $x \in \Gamma_{r}$ and $y \in \Gamma_{n-r}$, by (3.5) we clearly have

$$
\eta_{n}\left(\gamma \otimes r^{k} s^{l}\right)(x \times y, t)=\eta_{n}\left(\gamma \otimes r^{k} s^{l}\right)(x, t) \eta_{n}\left(\gamma \otimes r^{k} s^{l}\right)(y, t) .
$$

This immediately implies the formula. 
A similar argument gives that

$$
\varepsilon_{n}\left(\gamma \otimes r^{k} s^{l}\right)\left(x,\left(t_{1}, t_{2}\right)\right)=(-1)^{n} \prod_{c \in \Gamma_{*}}(-\gamma(c))^{l(\rho(c))} t_{1}^{n k} t_{2}^{n l},
$$

where $x$ is any element in the conjugacy class of type $\rho=(\rho(c))_{c \in \Gamma^{*}}$.

Formula (6.2) is equivalent to the following:

$$
\eta_{n}\left(\gamma \otimes r^{k} s^{l}\right)\left(c_{\rho},\left(t_{1}, t_{2}\right)\right)=\prod_{c \in \Gamma_{*}} \prod_{i \geq 1}\left(\gamma \otimes r^{k} s^{l}\right)\left(c,\left(t_{1}^{i}, t_{2}^{i}\right)^{m_{i}(\rho(c))} .\right.
$$

The following result allows us to extend the map from $\gamma \in \Gamma^{*}$ to $R\left(\Gamma_{n}\right)$.

Proposition 6.3. For any $\gamma \in R(\Gamma)$, we have

$$
\begin{aligned}
\sum_{n \geq 0} \operatorname{ch}\left(\eta_{n}\left(\gamma \otimes r^{k} s^{l}\right)\right) z^{n} & =\exp \left(\sum_{n \geq 1} \frac{1}{n} a_{-n}(\gamma)\left(r^{-k} s^{-l} z\right)^{n}\right) \\
\sum_{n \geq 0} \operatorname{ch}\left(\varepsilon_{n}\left(\gamma \otimes r^{k} s^{l}\right)\right) z^{n} & =\exp \left(\sum_{n \geq 1}(-1)^{n-1} \frac{1}{n} a_{-n}(\gamma)\left(r^{-k} s^{-l} z\right)^{n}\right) .
\end{aligned}
$$

Proof. It follows from the definition of ch, (6.1) and (6.4) that

$$
\begin{aligned}
& \sum_{n \geq 0} \operatorname{ch}\left(\eta_{n}\left(\gamma \otimes r^{k} s^{l}\right)\right) z^{n} \\
= & \sum_{\rho} Z_{\rho}^{-1} \prod_{c \in \Gamma_{*}} \prod_{i \geq 1} S\left(\gamma_{r^{i k} s^{i l}}(c)^{m_{i}(\rho(c))}\right) a_{-\rho(c)} z^{\|\rho\| \mid} r^{-k\|\rho\| \mid} s^{-l\|\rho\|} \\
= & \sum_{\rho} Z_{\rho}^{-1} \prod_{c \in \Gamma_{*}} \gamma(c)^{l(\rho(c))} a_{-\rho(c)}\left(r^{-k} s^{-l} z\right)^{\|\rho\|} \\
= & \prod_{c \in \Gamma_{*}}\left(\sum_{\lambda}\left(\zeta_{c}^{-1} \gamma(c)\right)^{l(\lambda)} z_{\lambda}^{-1} a_{-\lambda}(c)\left(r^{-k} s^{-l} z\right)^{|\lambda|}\right) \\
= & \exp \left(\sum_{n \geq 1} \frac{1}{n} \sum_{c \in \Gamma_{*}} \zeta_{c}^{-1} \gamma(c) a_{-n}(c)\left(r^{-k} s^{-l} z\right)^{n}\right) \\
= & \exp \left(\sum_{n \geq 1} \frac{1}{n} a_{-n}(\gamma)\left(r^{-k} s^{-l} z\right)^{n}\right) .
\end{aligned}
$$

Similarly we can prove (6.6) using the following identity:

$$
\begin{aligned}
\varepsilon_{n}\left(\gamma \otimes r^{k} s^{l}\right)(x) & =(-1)^{n} \prod_{c \in \Gamma_{*}} \prod_{i \geq 1}\left(-\gamma_{r^{i k} s^{i l}}(c)\right)^{m_{i}(\rho(c))} \\
& =\left(-r^{k} s^{l}\right)^{n} \prod_{c \in \Gamma_{*}} \prod_{i \geq 1}(-\gamma(c))^{m_{i}(\rho(c))} \\
& =\varepsilon_{n}(\gamma)(x) r^{n k} s^{n l} .
\end{aligned}
$$

The same argument as in the classical case (cf. [FJW1, FJW2 ) by using (3.6) and (3.7) will show that the proposition holds for a linear combination of simple characters such as $\gamma \otimes r^{k} s^{l}-\beta \otimes r^{k} s^{l}$, and thus it is true for any element $\gamma \otimes r^{k} s^{l}$, where $\gamma \in R(\Gamma)$. 
Comparing components we obtain

$$
\begin{aligned}
\operatorname{ch}\left(\eta_{n}\left(\gamma \otimes r^{k} s^{l}\right)\right) & =\sum_{\lambda} \frac{r^{-k n} s^{-l n}}{z_{\lambda}} a_{-\lambda}(\gamma), \\
\operatorname{ch}\left(\varepsilon_{n}\left(\gamma \otimes r^{k} s^{l}\right)\right) & =\sum_{\lambda} \frac{r^{-k n} s^{-l n}}{z_{\lambda}}(-1)^{|\lambda|-l(\lambda)} a_{-\lambda}(\gamma),
\end{aligned}
$$

where the sum runs over all partitions $\lambda$ of $n$.

Corollary 6.4. The formula (6.4) remains valid when $\gamma \otimes r^{k} s^{l}$ is replaced by any element $\xi \in R\left(\Gamma \times \mathbb{C}^{\times}\right)$. In particular $\eta_{n}(\xi)$ is self-dual provided that $\xi$ is invariant under the antipode $S$.

6.2. Isometry between $R_{\Gamma \times \mathbb{C}^{\times} \times \mathbb{C}^{\times}}$and $S_{\Gamma \times \mathbb{C}^{\times} \times \mathbb{C}^{\times}}$. The symmetric algebra $S_{\Gamma \times \mathbb{C}^{\times} \times \mathbb{C}^{\times}}=S_{\Gamma} \otimes \mathbb{C}\left[r^{ \pm 1}, s^{ \pm 1}\right]$ has the following Hopf algebra structure over $\mathbb{C}$. The multiplication is the usual one, and the comultiplication is given by

$$
\begin{aligned}
\Delta\left(r^{k} s^{l}\right) & =r^{k} s^{l} \otimes r^{k} s^{l}, \\
\Delta\left(a_{n}\left(\gamma \otimes r^{k} s^{l}\right)\right) & =a_{n}\left(\gamma \otimes r^{k} s^{l}\right) \otimes r^{n k} s^{n l}+r^{n k} s^{n l} \otimes a_{n}\left(\gamma \otimes r^{k} s^{l}\right),
\end{aligned}
$$

where $\gamma \in \Gamma^{*}$. The last formula is equivalent to the following:

$$
\Delta\left(a_{n}\left(c \otimes r^{k} s^{l}\right)\right)=a_{n}\left(c \otimes r^{k} s^{l}\right) \otimes r^{n k} s^{n l}+r^{n k} s^{n l} \otimes a_{n}\left(c \otimes r^{k} s^{l}\right),
$$

where $c \in \Gamma_{*}$. The antipode is given by

$$
\begin{aligned}
S\left(r^{k} s^{l}\right) & =r^{l} s^{k} \\
S\left(a_{n}\left(\gamma \otimes r^{k} s^{l}\right)\right) & =-a_{n}\left(\gamma \otimes r^{l} s^{k}\right) .
\end{aligned}
$$

The antipode commutes with the adjoint (dual) map $*$ :

$$
*^{2}=S^{2}=I d, \quad S *=* S .
$$

Recall that we have defined a Hopf algebra structure on $R_{\Gamma \times \mathbb{C} \times \times \mathbb{C} \times}$ in Section 2

Proposition 6.5. The characteristic map ch: $R_{\Gamma \times \mathbb{C}^{\times} \times \mathbb{C}^{\times}} \longrightarrow S_{\Gamma \times \mathbb{C}^{\times} \times \mathbb{C}^{\times}}$is an isomorphism of Hopf algebras.

Proof. This follows immediately from the definition of the comultiplication in both Hopf algebras (cf. (2.4) and (6.7)).

Recall that we have defined a bilinear form $\langle,\rangle_{\xi}$ on $R_{\Gamma \times \mathbb{C} \times \times \mathbb{C} \times}$ and a bilinear form on $S_{\Gamma \times \mathbb{C}^{\times} \times \mathbb{C}^{\times}}$denoted by $\langle,\rangle_{\xi}^{\prime}$, where $\xi$ is a self-dual class function. The following lemma is immediate from our definition of $\langle,\rangle_{\xi}^{\prime}$ and the comultiplication $\Delta$.

Lemma 6.6. The bilinear form $\langle,\rangle_{\xi}^{\prime}$ on $S_{\Gamma \times \mathbb{C}^{\times} \times \mathbb{C}^{\times}}$can be characterized by the following two properties:

1). $\left\langle a_{-n}\left(\beta \otimes r^{k_{1}} s^{l_{1}}\right), a_{-m}\left(\gamma \otimes r^{k_{2}} s^{l_{2}}\right)\right\rangle_{\xi}^{\prime}=\delta_{n, m} r^{n\left(k_{2}-k_{1}\right)} s^{n\left(l_{2}-l_{1}\right)}\langle\beta, \gamma\rangle_{\xi}^{\prime}, \quad \beta, \gamma \in$ $\Gamma^{*}, k_{1}, k_{2}, l_{1}, l_{2} \in \mathbb{Z}$.

2). $\langle f g, h\rangle_{\xi}^{\prime}=\langle f \otimes g, \Delta h\rangle_{\xi}^{\prime}$, where $f, g, h \in S_{\Gamma \times \mathbb{C}^{\times} \times \mathbb{C}^{\times}}$, and the bilinear form on $S_{\Gamma \times \mathbb{C}^{\times} \times \mathbb{C}^{\times}} \otimes S_{\Gamma \times \mathbb{C}^{\times} \times \mathbb{C}^{\times}}$, is induced from $\langle,\rangle_{\xi}^{\prime}$ on $S_{\Gamma \times \mathbb{C}^{\times} \times \mathbb{C}^{\times}}$.

Theorem 6.7. The characteristic map is an isometry from $\left(R_{\Gamma \times \mathbb{C}^{\times} \times \mathbb{C}^{\times}},\langle,\rangle_{\xi}\right)$ to $\left(S_{\Gamma \times \mathbb{C}^{\times} \times \mathbb{C}^{\times}},\langle,\rangle_{\xi}^{\prime}\right)$. 
Proof. By Corollary 6.4, the character value of $\eta_{n}(\xi)$ at an element $x$ of type $\rho$ is

$$
\eta_{n}(\xi)(x)=\prod_{c \in \Gamma_{*}} \prod_{i \geq 1} \xi_{r^{i}, s^{i}}(c)^{m_{i}(\rho(c))} .
$$

Thus it follows from the definition that

$$
\begin{aligned}
\left\langle\sigma_{\rho \otimes r^{k_{1}} s^{l_{1}}}, \sigma_{\left.\rho^{\prime} \otimes r^{k_{2}} s^{l_{2}}\right\rangle_{\xi}}\right. & =\sum_{\mu \in \mathcal{P}_{n}\left(\Gamma_{*}\right)} Z_{\mu}^{-1} r^{n\left(k_{2}-k_{1}\right)} s^{n\left(l_{2}-l_{1}\right)} \xi_{r, s}\left(c_{\mu}\right) \sigma_{\rho}\left(c_{\mu}\right) \sigma_{\rho^{\prime}}\left(c_{\mu}\right) \\
& =\delta_{\rho, \rho^{\prime}} Z_{\rho}^{-1} r^{n\left(k_{2}-k_{1}\right)} s^{n\left(l_{2}-l_{1}\right)} \xi\left(c_{\rho}\right) Z_{\rho} Z_{\rho} \\
& =\delta_{\rho, \rho^{\prime}} Z_{\rho} r^{n\left(k_{2}-k_{1}\right)} s^{n\left(l_{2}-l_{1}\right)} \prod_{c \in \Gamma_{*}} \prod_{i \geq 1} \xi_{q^{i}}(c)^{m_{i}(\rho(c))}
\end{aligned}
$$

By Lemma 6.1 and the formula (5.6), we see that

$$
\begin{aligned}
\left\langle\sigma_{\rho \otimes r^{k_{1}} s^{l_{1}}}, \sigma_{\rho^{\prime} \otimes r^{k_{2}} s^{l_{2}}}\right\rangle_{\xi} & =\left\langle a_{-\rho \otimes r^{k_{1}} s^{l_{1}}}, a_{-\rho^{\prime} \otimes r^{k_{2}} s_{2} l_{2}}\right\rangle_{\xi}^{\prime} \\
& =\left\langle\operatorname{ch}\left(\sigma_{\rho \otimes r^{k_{1}} s^{l_{1}}}\right), \operatorname{ch}\left(\sigma_{\rho^{\prime} \otimes r^{k_{2}} s_{2}}\right)\right\rangle_{\xi}^{\prime} .
\end{aligned}
$$

Since $\sigma_{\rho \otimes r^{k} s^{l}}, \rho \in \mathcal{P}\left(\Gamma_{*}\right)$ form a $\mathbb{C}$-basis of $R_{\Gamma \times \mathbb{C}^{\times} \times \mathbb{C}^{\times}}$, we have shown that ch : $R_{\Gamma \times \mathbb{C}^{\times} \times \mathbb{C}^{\times}} \longrightarrow S_{\Gamma \times \mathbb{C}^{\times} \times \mathbb{C}^{\times}}$is an isometry.

From now on we will not distinguish the bilinear form $\langle,\rangle_{\xi}$ on $R_{\Gamma \times \mathbb{C}^{\times} \times \mathbb{C}^{\times}}$from the bilinear form $\langle,\rangle_{\xi}^{\prime}$ on $S_{\Gamma \times \mathbb{C}^{\times} \times \mathbb{C}^{\times}}$.

\section{Two-Parameter quantum Vertex operators and $R_{\Gamma \times \mathbb{C}^{\times} \times \mathbb{C}^{\times}}$}

7.1. Two-parameter vertex operators and Heisenberg algebras in $\mathcal{F}_{\Gamma \times \mathbb{C}^{\times} \times \mathbb{C}^{\times}}$. Let $\mathbb{K}=\mathbb{Q}(r, s)$ denote a field of rational functions with two parameters $r, s(r \neq \pm s)$. Let $Q$ be an integral lattice with the basis $\alpha_{i}, i=0,1, \ldots, n-1$ endowed with a symmetric bilinear form. Let $\epsilon: Q \times Q \longrightarrow \mathbb{K}^{\times}$be the 2-cocycle such that

$$
\begin{aligned}
& \epsilon(\alpha+\beta, \gamma)=\epsilon(\alpha, \beta) \epsilon(\beta, \gamma) \\
& \epsilon(\alpha, \beta+\gamma)=\epsilon(\alpha, \beta) \epsilon(\alpha, \gamma)
\end{aligned}
$$

We fix $\epsilon$ by choosing directly

$$
\epsilon\left(\alpha_{i}, \alpha_{j}\right)=\left\{\begin{array}{cc}
\left(-r_{i} s_{i}\right)^{\frac{a_{i j}}{2}}, & i>j \\
(r s)^{\frac{1}{2}}, & i=j \\
1, & i<j
\end{array}\right.
$$

and extend to $Q \times Q$.

Let $\xi$ be a self-dual virtual character in $R_{\Gamma \times \mathbb{C}^{\times} \times \mathbb{C}^{\times}}$. Recall that the lattice $R_{\mathbb{Z}}(\Gamma)$ is a $\mathbb{Z}$-lattice under the bilinear form $\langle,\rangle_{\xi}^{1}$; here the superscript means $r=s^{-1}=1$. For our purpose we will always associate a 2-cocycle $\epsilon$ as in the previous subsection to the integral lattice $\left(R_{\mathbb{Z}}(\Gamma),\langle,\rangle_{\xi}^{1}\right)$ (and its sublattices).

Let $\mathbb{C}\left[R_{\mathbb{Z}}(\Gamma)\right]$ be the group algebra generated by $e^{\gamma}, \gamma \in R_{\mathbb{Z}}(\Gamma)$. We introduce two special operators acting on $\mathbb{C}\left[R_{\mathbb{Z}}(\Gamma)\right]$ : A ( $\epsilon$-twisted) multiplication operator $e^{\alpha}$ defined by

$$
e^{\alpha} \cdot e^{\beta}=\epsilon(\alpha, \beta) e^{\alpha+\beta}, \quad \alpha, \beta \in R_{\mathbb{Z}}(\Gamma),
$$


and a differentiation operator $\partial_{\alpha}$ given by

$$
\partial_{\alpha} e^{\beta}=\langle\alpha, \beta\rangle_{\xi}^{1} e^{\beta}, \quad \alpha, \beta \in R_{\mathbb{Z}}(\Gamma) .
$$

These two operators are then extended linearly to the space

$$
\mathcal{F}_{\Gamma \times \mathbb{C}^{\times} \times \mathbb{C}^{\times}}=R_{\Gamma \times \mathbb{C}^{\times} \times \mathbb{C}^{\times}} \otimes \mathbb{C}\left[R_{\mathbb{Z}}(\Gamma)\right]
$$

by letting them act on $R_{\Gamma \times \mathbb{C} \times \times \mathbb{C} \times}$ trivially.

We define the Hopf algebra structure on $\mathbb{C}\left[R_{\mathbb{Z}}(\Gamma)\right]$ and extend it to $\mathcal{F}_{\Gamma \times \mathbb{C} \times} \times \mathbb{C}^{\times}$ as follows:

$$
\Delta\left(e^{\alpha}\right)=e^{\alpha} \otimes e^{\alpha}, \quad S\left(e^{\alpha}\right)=e^{-\alpha} .
$$

The bilinear form $\langle,\rangle_{\xi}^{r, s}$ on $R_{\Gamma \times \mathbb{C}^{\times} \times \mathbb{C}^{\times}}$is extended to $\mathcal{F}_{\Gamma \times \mathbb{C}^{\times} \times \mathbb{C}^{\times}}$by

$$
\left\langle e^{\alpha}, e^{\beta}\right\rangle_{\xi}=\delta_{\alpha, \beta} .
$$

With respect to this extended bilinear form we have the $*$-action (adjoint action) on the operators $e^{\alpha}$ and $\partial_{\alpha}$ :

$$
\left(e^{\alpha}\right)^{*}=e^{-\alpha}, \quad\left(z^{\partial_{\alpha}}\right)^{*}=z^{-\partial_{\alpha}} .
$$

For each $k \in \mathbb{Z}$, we introduce the group-theoretic operators $H_{ \pm n}\left(\gamma \otimes r^{k} s^{l}\right)$, $E_{ \pm n}\left(\gamma \otimes r^{k} s^{l}\right), \gamma \in R(\Gamma), n>0$ as the following compositions of maps:

$$
\begin{aligned}
& H_{-n}\left(\gamma \otimes r^{k} s^{l}\right) \quad: \quad R\left(\Gamma_{m} \times \mathbb{C}^{\times} \times \mathbb{C}^{\times}\right) \stackrel{\eta_{n}\left(\gamma \otimes r^{k} s^{l}\right) \otimes}{\longrightarrow} R\left(\Gamma_{n} \times \mathbb{C}^{\times} \times \mathbb{C}^{\times}\right) \otimes R\left(\Gamma_{m} \times \mathbb{C}^{\times} \times \mathbb{C}^{\times}\right) \\
& \stackrel{\text { Ind } \otimes m_{\mathbb{C} \times}}{\longrightarrow} R\left(\Gamma_{n+m} \times \mathbb{C}^{\times} \times \mathbb{C}^{\times}\right), \\
& E_{-n}\left(\gamma \otimes r^{k} s^{l}\right): \quad R\left(\Gamma_{m} \times \mathbb{C}^{\times} \times \mathbb{C}^{\times}\right) \stackrel{\varepsilon_{n}\left(\gamma \otimes r^{k} s^{l}\right) \otimes}{\longrightarrow} R\left(\Gamma_{n} \times \mathbb{C}^{\times} \times \mathbb{C}^{\times}\right) \otimes R\left(\Gamma_{m} \times \mathbb{C}^{\times} \times \mathbb{C}^{\times}\right) \\
& \stackrel{\text { Ind } \otimes m_{\mathbb{C}^{\times}}}{\longrightarrow} R\left(\Gamma_{n+m} \times \mathbb{C}^{\times} \times \mathbb{C}^{\times}\right), \\
& E_{n}\left(\gamma \otimes r^{k} s^{l}\right) \quad: \quad R\left(\Gamma_{m} \times \mathbb{C}^{\times} \times \mathbb{C}^{\times}\right) \stackrel{\operatorname{Res}}{\longrightarrow} R\left(\Gamma_{n}\right) \otimes R\left(\Gamma_{m-n} \times \mathbb{C}^{\times} \times \mathbb{C}^{\times}\right) \\
& \left\langle\varepsilon_{n}\left(\gamma \otimes r^{k} s^{l}\right),\right\rangle_{\xi} R\left(\Gamma_{m-n} \times \mathbb{C}^{\times} \times \mathbb{C}^{\times}\right), \\
& H_{n}\left(\gamma \otimes r^{k} s^{l}\right) \quad: \quad R\left(\Gamma_{m} \times \mathbb{C}^{\times} \times \mathbb{C}^{\times}\right) \stackrel{\operatorname{Res}}{\longrightarrow} R\left(\Gamma_{n}\right) \otimes R\left(\Gamma_{m-n} \times \mathbb{C}^{\times} \times \mathbb{C}^{\times}\right) \\
& \left\langle\eta_{n}\left(\stackrel{\gamma \otimes q^{k}}{\longrightarrow}\right),\right\rangle_{\xi} R\left(\Gamma_{m-n} \times \mathbb{C}^{\times} \times \mathbb{C}^{\times}\right),
\end{aligned}
$$

where Res and Ind are the restriction and induction functors in $R_{\Gamma}=\bigoplus_{n \geq 0} R\left(\Gamma_{n}\right)$.

We introduce their generating functions in a formal variable $z$ :

$$
\begin{aligned}
& H_{ \pm}\left(\gamma \otimes r^{k} s^{l}, z\right)=\sum_{n \geq 0} H_{\mp n}\left(\gamma \otimes r^{k} s^{l}\right) z^{ \pm n} \\
& E_{ \pm}\left(\gamma \otimes r^{k} s^{l}, z\right)=\sum_{n \geq 0} E_{\mp n}\left(\gamma \otimes r^{k} s^{l}\right)(-z)^{ \pm n} .
\end{aligned}
$$

We now define the vertex operators $Y_{n}^{ \pm}\left(\gamma \otimes r^{k} s^{l}, a, b\right), \gamma \in \Gamma^{*}, k, l, a, b \in \mathbb{Z}$, $n \in \mathbb{Z}+\langle\gamma, \gamma\rangle_{\xi}^{1} / 2$ as follows:

$$
\begin{aligned}
Y^{+}\left(\gamma \otimes r^{k} s^{l}, a, b, z\right) & =\sum_{n \in \mathbb{Z}+\langle\gamma, \gamma\rangle_{\xi}^{1} / 2} Y_{n}^{+}\left(\gamma \otimes r^{k} s^{l}, a, b\right) z^{-n-\langle\gamma, \gamma\rangle_{\xi}^{1} / 2} \\
& =H_{+}\left(\gamma \otimes r^{k} s^{l}, z\right) E_{-}\left(\gamma \otimes r^{k-a} s^{l-b}, z\right) e^{\gamma}\left(r^{-k} s^{-l} z\right)^{\partial_{\gamma}}
\end{aligned}
$$




$$
\begin{aligned}
Y^{-}\left(\gamma \otimes r^{k} s^{l}, a, b, z\right) & =\left(Y^{+}\left(\gamma \otimes r^{k} s^{l}, a, b, z^{-1}\right)\right)^{*} \\
& =\sum_{n \in \mathbb{Z}+\langle\gamma, \gamma\rangle_{\xi}^{1} / 2} Y_{n}^{-}\left(\gamma \otimes r^{k} s^{l}, a, b\right) z^{-n-\langle\gamma, \gamma\rangle_{\xi}^{1} / 2} \\
& =E_{+}\left(\gamma \otimes r^{k-a} s^{l-b}, z\right) H_{-}\left(\gamma \otimes r^{k} s^{l}, z\right) e^{-\gamma}\left(r^{-k} s^{-l} z\right)^{-\partial_{\gamma}} .
\end{aligned}
$$

One easily sees that the operators $Y_{n}^{ \pm}\left(\gamma \otimes r^{k} s^{l}, a, b\right)$ are well-defined operators acting on the space $\mathcal{F}_{\Gamma \times \mathbb{C}^{\times} \times \mathbb{C}^{\times} \text {. }}$

We extend the $\mathbb{Z}_{+}$-gradation on $R_{\Gamma \times \mathbb{C}^{\times} \times \mathbb{C}^{\times}}$to a $\frac{1}{2}\langle\gamma, \gamma\rangle_{\xi}^{1}+\mathbb{Z}_{+}$-gradation on $\mathcal{F}_{\Gamma \times \mathbb{C}^{\times} \times \mathbb{C}^{\times}}$by letting

$$
\operatorname{deg} a_{-n}\left(\gamma \otimes r^{k} s^{l}\right)=n, \quad \operatorname{deg} e^{\gamma}=\frac{1}{2}\langle\gamma, \gamma\rangle_{\xi}^{1} .
$$

We denote by $\bar{R}_{\Gamma \times \mathbb{C} \times \times \mathbb{C} \times}$ the subalgebra of $R_{\Gamma \times \mathbb{C} \times \times \mathbb{C} \times}$ excluding the generators $a_{n}\left(\gamma_{0}\right), n \in \mathbb{Z}^{\times}$. The bilinear form $\langle,\rangle_{\xi}$ on

$$
\overline{\mathcal{F}}_{\Gamma \times \mathbb{C}^{\times} \times \mathbb{C}^{\times}}=\bar{R}_{\Gamma \times \mathbb{C}^{\times} \times \mathbb{C}^{\times}} \otimes \bar{R}_{\mathbb{Z}}(\Gamma)
$$

will be the restriction of $\langle,\rangle_{\xi}$ on $\mathcal{F}_{\Gamma \times \mathbb{C} \times \times \mathbb{C} \times}$ to $\overline{\mathcal{F}}_{\Gamma \times \mathbb{C} \times \times \mathbb{C} \times}$.

We define $\widetilde{a}_{-n}\left(\gamma \otimes r^{k} s^{l}\right), n>0$ to be a map from $R_{\Gamma \times \mathbb{C}^{\times} \times \mathbb{C}^{\times}}$to itself by the following composition:

$$
\begin{gathered}
R\left(\Gamma_{m} \times \mathbb{C}^{\times} \times \mathbb{C}^{\times}\right) \stackrel{\sigma_{n}\left(\gamma \otimes r^{k} s^{l}\right) \otimes}{\longrightarrow} R\left(\Gamma_{n} \times \mathbb{C}^{\times} \times \mathbb{C}^{\times}\right) \otimes R\left(\Gamma_{m} \times \mathbb{C}^{\times} \times \mathbb{C}^{\times}\right) \\
\stackrel{\operatorname{Ind} \otimes m_{\mathbb{C}^{\times}}}{\longrightarrow} R\left(\Gamma_{n+m} \times \mathbb{C}^{\times} \times \mathbb{C}^{\times}\right) .
\end{gathered}
$$

We also define $\widetilde{a}_{n}\left(\gamma \otimes r^{k} s^{l}\right), n>0$ to be a map from $R_{\Gamma \times \mathbb{C}^{\times} \times \mathbb{C}^{\times}}$to itself as the composition

$$
\begin{aligned}
& R\left(\Gamma_{m} \times \mathbb{C}^{\times} \times \mathbb{C}^{\times}\right) \stackrel{\operatorname{Res} 1}{\longrightarrow} R\left(\Gamma_{n} \times \mathbb{C}^{\times} \times \mathbb{C}^{\times}\right) \otimes R\left(\Gamma_{m-n} \times \mathbb{C}^{\times} \times \mathbb{C}^{\times}\right) \\
& \left\langle\sigma_{n}\left(\gamma \otimes r^{k} s^{l}\right), \cdot\right\rangle_{\xi}^{r, s} R\left(\Gamma_{m-n} \times \mathbb{C}^{\times} \times \mathbb{C}^{\times}\right) .
\end{aligned}
$$

Proposition 7.1. The operators $\widetilde{a}_{n}(\gamma), \gamma \in \Gamma^{*}, n \in \mathbb{Z}^{\times}$satisfy the Heisenberg algebra relations (5.1) with $C=1$.

Proof. This is similarly proved as for the classical setting in [W].

7.2. Group-theoretic interpretation of vertex operators. To compare the vertex operators $Y^{ \pm}\left(\gamma \otimes r^{k} s^{l}, a, b, z\right)$ with the familiar vertex operators acting in the Fock space we introduce the space

$$
V_{\Gamma \times \mathbb{C}^{\times} \times \mathbb{C}^{\times}}=S_{\Gamma \times \mathbb{C}^{\times} \times \mathbb{C}^{\times}} \otimes \mathbb{C}\left[R_{\mathbb{Z}}(\Gamma)\right] .
$$

We extend the bilinear form $\langle,\rangle_{\xi}^{r, s}$ in $S_{\Gamma \times \mathbb{C}^{\times} \times \mathbb{C}^{\times}}$to the space $V_{\Gamma \times \mathbb{C}^{\times} \times \mathbb{C}^{\times}}$and also extend the $\mathbb{Z}_{+}$-gradation on $S_{\Gamma \times \mathbb{C}^{\times} \times \mathbb{C}^{\times}}$to a $\frac{1}{2} \mathbb{Z}_{+}$-gradation on $V_{\Gamma}$.

We extend the characteristic map to the map

$$
\text { ch: } \mathcal{F}_{\Gamma \times \mathbb{C}^{\times} \times \mathbb{C}^{\times}} \longrightarrow V_{\Gamma \times \mathbb{C}^{\times} \times \mathbb{C}^{\times}}
$$

by identity on $R_{\mathbb{Z}}(\Gamma)$. Then Proposition 6.5 and Theorem 6.7 imply that we have an isometric isomorphism of Hopf algebras. We can now identify the operators 
from the previous subsections with the operators constructed from the Heisenberg algebra.

Theorem 7.2. For any $\gamma \in R(\Gamma)$ and $k \in \mathbb{Z}$, we have

$$
\begin{aligned}
\operatorname{ch}\left(H_{+}\left(\gamma \otimes r^{k} s^{l}, z\right)\right) & =\exp \left(\sum_{n \geq 1} \frac{1}{n} a_{-n}(\gamma)\left(r^{-k} s^{-l} z\right)^{n}\right) \\
c h\left(E_{+}\left(\gamma \otimes r^{k} s^{l}, z\right)\right) & =\exp \left(-\sum_{n \geq 1} \frac{1}{n} a_{-n}(\gamma)\left(r^{-k} s^{-l} z\right)^{n}\right) \\
c h\left(H_{-}\left(\gamma \otimes r^{k} s^{l}, z\right)\right) & =\exp \left(\sum_{n \geq 1} \frac{1}{n} a_{n}(\gamma)\left(r^{-k} s^{-l} z\right)^{-n}\right) \\
c h\left(E_{-}\left(\gamma \otimes r^{k} s^{l}, z\right)\right) & =\exp \left(-\sum_{n \geq 1} \frac{1}{n} a_{n}(\gamma)\left(r^{-k} s^{-l} z\right)^{-n}\right) .
\end{aligned}
$$

Proof. The first and second identities have been essentially established in Proposition 6.3 together with Lemma 6.1 where the components are viewed as operators acting on $R_{\Gamma \times \mathbb{C}^{\times} \times \mathbb{C}^{\times}}$or $S_{\Gamma \times \mathbb{C}^{\times} \times \mathbb{C}^{\times}}$. Note that $a_{n}\left(\gamma \otimes r^{k} s^{l}\right)=a_{n}(\gamma) r^{k n} s^{l n}$.

We observe from the definition that the adjoint $*$-actions of $E_{+}\left(\gamma \otimes r^{k} s^{l}, z\right)$ and $H_{-}\left(\gamma \otimes r^{k} s^{l}, z\right)$ with respect to the bilinear form $\langle,\rangle_{\xi}^{r, s}$ are $E_{-}\left(\gamma \otimes r^{k} s^{l}, z^{-1}\right)$ and $H_{-}\left(\gamma \otimes r^{k} s^{l}, z^{-1}\right)$ respectively. The third and fourth identities are obtained by applying the adjoint action $*$ to the first two identities.

Remark 7.3. Replacing $\gamma$ by $-\gamma$ in (17.5) and (7.7) we obtain the equivalent formulas (7.6) and (7.8) respectively.

Applying the characteristic map to the vertex operators $Y^{ \pm}(\gamma, a, b, z)$, we obtain the following group-theoretical explanation of vertex operators acting on the Fock space $\mathcal{F}_{\Gamma \times \mathbb{C}^{\times} \times \mathbb{C}^{\times}}$.

Theorem 7.4. For any $\gamma \in R_{\Gamma}$ and $k \in \mathbb{Z}$, we have

$$
\begin{aligned}
& Y^{+}(\gamma, a, b, z)=\exp \left(\sum_{n \geq 1} \frac{1}{n} \widetilde{a}_{-n}(\gamma) z^{n}\right) \exp \left(-\sum_{n \geq 1} \frac{1}{n} \widetilde{a}_{n}(\gamma) r^{-a n} s^{-b n} z^{-n}\right) e^{\gamma} z^{\partial_{\gamma}} \\
& =\operatorname{ch}\left(H_{+}(\gamma, z)\right) \operatorname{ch}\left(S\left(H_{+}\left(\gamma \otimes r^{a} s^{b}, z^{-1}\right)^{*}\right)\right) e^{\gamma} z^{\partial_{\gamma}} \\
& Y^{-}(\gamma, a, b, z)=\exp \left(-\sum_{n \geq 1} \frac{1}{n} \widetilde{a}_{-n}(\gamma) r^{a n} s^{b n} z^{n}\right) \exp \left(\sum_{n \geq 1} \frac{1}{n} \widetilde{a}_{n}(\gamma) z^{-n}\right) e^{-\gamma} z^{-\partial_{\gamma}} \\
& =\operatorname{ch}\left(S\left(H_{+}\left(\gamma \otimes r^{a} s^{b}, z^{-1}\right)\right)\right) \operatorname{ch}\left(H_{+}(\gamma, z)^{*}\right) e^{-\gamma} z^{-\partial_{\gamma}}
\end{aligned}
$$

We note that for $\gamma \in \Gamma^{*}, k, l \in \mathbb{Z}$,

$$
Y^{ \pm}\left(\gamma \otimes r^{k} s^{l}, a, b, z\right)=Y^{ \pm}\left(\gamma, a, b, r^{-k} s^{-l} z\right)
$$


It follows from Theorem 7.4 that

$$
\begin{aligned}
& \operatorname{ch}\left(Y^{+}(\gamma, a, b, z)\right)=X^{+}(\gamma, a, b, z) \\
& =\exp \left(\sum_{n \geq 1} \frac{1}{n} a_{-n}(\gamma) z^{n}\right) \\
& \quad \times \exp \left(-\sum_{n \geq 1} \frac{1}{n} a_{n}(\gamma) r^{-a n} s^{-b n} z^{-n}\right) e^{\gamma} z^{\partial_{\gamma}} \\
& \operatorname{ch}\left(Y^{-}(\gamma, a, b, z)\right)=X^{-}(\gamma, a, b, z) \\
& =\exp \left(-\sum_{n \geq 1} \frac{1}{n} a_{-n}(\gamma) r^{a n} s^{b n} z^{n}\right) \\
& \quad \times \exp \left(\sum_{n \geq 1} \frac{1}{n} a_{n}(\gamma) z^{-n}\right) e^{-\gamma} z^{-\partial_{\gamma}} .
\end{aligned}
$$

When $r=s^{-1}=q$, they specialize to the vertex operators $Y^{ \pm}(\gamma, k, z)$ studied in FJW2].

Under the new variable (by identifying $a_{i}(n)$ with $\widetilde{a_{i}}(n)$ ) we obtain that

$$
\begin{aligned}
& X^{+}\left(\gamma_{i} \otimes s^{-b}, a, b, z\right)=\exp \left(\sum_{n \geq 1} \frac{a_{i}(-n)}{[n]} s^{-b n} z^{n}\right) \exp \left(-\sum_{n \geq 1} \frac{a_{i}(n)}{[n]} r^{-a n} z^{-n}\right) e^{\gamma} z^{\partial_{\gamma}} \\
& X^{-}\left(\gamma_{i} \otimes r^{-a}, k, z\right)=\exp \left(-\sum_{n \geq 1} \frac{a_{i}(-n)}{[n]} s^{b n} z^{n}\right) \exp \left(\sum_{n \geq 1} \frac{a_{i}(n)}{[n]} r^{a n} z^{-n}\right) e^{-\gamma} z^{-\partial_{\gamma}}
\end{aligned}
$$

\section{Basic Representations And the MCKay CORREspondence}

8.1. Two-parameter quantum toroidal algebras. In this subsection we define the two-parameter quantum toroidal algebras $U_{r, s}(\widehat{\widehat{\mathfrak{g}}})$ of simply laced type $A, D$ or $E$. In particular the two-parameter quantum toroidal algebra contains a special subalgebra: the two-parameter quantum affine algebras $U_{r, s}(\widehat{\mathfrak{g}})(\operatorname{cf}$. [HRZ, HZ, Z] $)$.

Let $\left(A_{i j}\right)$ be the two-parameter quantum Cartan $(N+1) \times(N+1)$ - Martix (cf. [HZ, Z]). For type $A_{n}^{(1)}$, we have

$$
A_{i j}=\left(\begin{array}{cccccc}
r s^{-1} & r^{-1} & 1 & \ldots & 1 & s \\
s & r s^{-1} & r^{-1} & \ldots & 1 & 1 \\
\ldots & \ldots & \ldots & \ldots & \ldots & \ldots \\
1 & 1 & 1 & \ldots & r s^{-1} & r^{-1} \\
r^{-1} & 1 & 1 & \ldots & s & r s^{-1}
\end{array}\right)
$$

Definition 8.1. The two-parameter quantum toroidal algebra $U_{r, s}(\widehat{\widehat{\mathfrak{g}}})$ is an associative algebra over $\mathbb{K}$ generated by the elements $x_{i}^{ \pm}(k), a_{i}(m), \omega_{i}^{ \pm 1}, \omega_{i}^{ \pm 1}, \gamma^{ \pm \frac{1}{2}}$, $\gamma^{\prime \pm \frac{1}{2}}, D^{ \pm 1}, D^{\prime \pm 1}\left(0 \leq i \leq N, k, k^{\prime} \in \mathbb{Z}, m, m^{\prime} \in \mathbb{Z} \backslash\{0\}\right)$, subject to the following defining relations. 
(D1) $\gamma^{ \pm \frac{1}{2}}, \gamma^{\prime \pm \frac{1}{2}}$ are central with $\gamma \gamma^{\prime}=r s, \omega_{i} \omega_{i}^{-1}=\omega_{j}^{\prime} \omega_{j}^{\prime-1}=1(i, j \in I)$, and

$$
\begin{aligned}
& {\left[\omega_{i}^{ \pm 1}, \omega_{j}^{ \pm 1}\right]=\left[\omega_{i}^{ \pm 1}, D^{ \pm 1}\right]=\left[\omega_{j}^{\prime \pm 1}, D^{ \pm 1}\right]=\left[\omega_{i}^{ \pm 1}, D^{ \pm 1}\right]=0} \\
& =\left[\omega_{i}^{ \pm 1}, \omega_{j}^{\prime \pm 1}\right]=\left[\omega_{j}^{\prime \pm 1}, D^{\prime \pm 1}\right]=\left[D^{\prime \pm 1}, D^{ \pm 1}\right]=\left[\omega_{i}^{\prime \pm 1}, \omega_{j}^{\prime \pm 1}\right] .
\end{aligned}
$$

$$
\left[a_{i}(m), a_{j}\left(m^{\prime}\right)\right]=\delta_{m+m^{\prime}, 0} \frac{(r s)^{\frac{|m|}{2}}\left(A_{i i}^{\frac{m a_{i j}}{2}}-A_{i i}^{-\frac{m a_{i j}}{2}}\right)}{|m|(r-s)} \cdot \frac{\gamma^{|m|}-\gamma^{\prime|m|}}{r-s} \text {. }
$$

$$
\left[a_{i}(m), \omega_{j}^{ \pm 1}\right]=\left[a_{i}(m), \omega_{j}^{\prime \pm 1}\right]=0 .
$$

$$
\begin{array}{rlrl}
D x_{i}^{ \pm}(k) D^{-1}=r^{k} x_{i}^{ \pm}(k), & D^{\prime} x_{i}^{ \pm}(k) D^{\prime-1} & =s^{k} x_{i}^{ \pm}(k), \\
D a_{i}(m) D^{-1}=r^{m} a_{i}(m), & D^{\prime} a_{i}(m) D^{\prime-1}=s^{m} a_{i}(m) .
\end{array}
$$

$$
\omega_{i} x_{j}^{ \pm}(k) \omega_{i}^{-1}=A_{j i}^{ \pm 1} x_{j}^{ \pm}(k), \quad \omega_{i}^{\prime} x_{j}^{ \pm}(k) \omega_{i}^{\prime-1}=A_{i j}^{\mp 1} x_{j}^{ \pm}(k) .
$$

$$
\left[a_{i}(m), x_{j}^{ \pm}(k)\right]= \pm \frac{(r s)^{\frac{|m|}{2}}\left(\left(r s^{-1}\right)^{\frac{m a_{i j}}{2}}-\left(r s^{-1}\right)^{-\frac{m a_{i j}}{2}}\right)}{m(r-s)}
$$

$$
\cdot \gamma^{ \pm \frac{m}{2}} x_{j}^{ \pm}(m+k), \quad \text { for } \quad m<0,
$$

$$
\left[a_{i}(m), x_{j}^{ \pm}(k)\right]= \pm \frac{(r s)^{\frac{|m|}{2}}\left(\left(r s^{-1}\right)^{\frac{m a_{i j}}{2}}-\left(r s^{-1}\right)^{-\frac{m a_{i j}}{2}}\right)}{m(r-s)}
$$

$$
\begin{gathered}
\cdot \gamma^{\prime \pm \frac{m}{2}} x_{j}^{ \pm}(m+k), \quad \text { for } m>0 . \\
x_{i}^{ \pm}(k+1) x_{j}^{ \pm}\left(k^{\prime}\right)-A_{j i}^{ \pm 1} x_{j}^{ \pm}\left(k^{\prime}\right) x_{i}^{ \pm}(k+1) \\
=-\left(A_{j i} A_{i j}^{-1}\right)^{ \pm \frac{1}{2}}\left(x_{j}^{ \pm}\left(k^{\prime}+1\right) x_{i}^{ \pm}(k)-A_{i j}^{ \pm 1} x_{i}^{ \pm}(k) x_{j}^{ \pm}\left(k^{\prime}+1\right)\right) . \\
{\left[x_{i}^{+}(k), x_{j}^{-}\left(k^{\prime}\right)\right]=\frac{\delta_{i j}}{r-s}\left(\gamma^{\prime-k} \gamma^{-\frac{k+k^{\prime}}{2}} \omega_{i}\left(k+k^{\prime}\right)-\gamma^{k^{\prime}} \gamma^{\prime \frac{k+k^{\prime}}{2}} \omega_{i}^{\prime}\left(k+k^{\prime}\right)\right),}
\end{gathered}
$$

where $\omega_{i}(m), \omega_{i}^{\prime}(-m)\left(m \in \mathbb{Z}_{\geq 0}\right)$ with $\omega_{i}(0)=\omega_{i}$ and $\omega_{i}^{\prime}(0)=\omega_{i}^{\prime}$ are defined by

$$
\begin{gathered}
\sum_{m=0}^{\infty} \omega_{i}(m) z^{-m}=\omega_{i} \exp \left((r-s) \sum_{\ell=1}^{\infty} a_{i}(\ell) z^{-\ell}\right), \\
\sum_{m=0}^{\infty} \omega_{i}^{\prime}(-m) z^{m}=\omega_{i}^{\prime} \exp \left(-(r-s) \sum_{\ell=1}^{\infty} a_{i}(-\ell) z^{\ell}\right),
\end{gathered}
$$

with $\omega_{i}(-m)=0$ and $\omega_{i}^{\prime}(m)=0, \forall m>0$.

$$
\begin{gathered}
\operatorname{Sym}_{m_{1}, \cdots, m_{n}} \sum_{k=0}^{n=1-a_{i j}}(-1)^{k}\left(r_{i} s_{i}\right)^{ \pm \frac{k(k-1)}{2}}\left[\begin{array}{c}
1-a_{i j} \\
k
\end{array}\right]_{ \pm i} x_{i}^{ \pm}\left(m_{1}\right) \cdots x_{i}^{ \pm}\left(m_{k}\right) x_{j}^{ \pm}(\ell) \\
\times x_{i}^{ \pm}\left(m_{k+1}\right) \cdots x_{i}^{ \pm}\left(m_{n}\right)=0, \quad a_{i j}<0, \quad 0 \leq j<i<N,
\end{gathered}
$$

$$
\begin{gathered}
\operatorname{Sym}_{m_{1}, \cdots, m_{n}} \sum_{k=0}^{n=1-a_{i j}}(-1)^{k}\left(r_{i} s_{i}\right)^{\mp \frac{k(k-1)}{2}}\left[\begin{array}{c}
1-a_{i j} \\
k
\end{array}\right]_{\mp i} x_{i}^{ \pm}\left(m_{1}\right) \cdots x_{i}^{ \pm}\left(m_{k}\right) x_{j}^{ \pm}(\ell) \\
\times x_{i}^{ \pm}\left(m_{k+1}\right) \cdots x_{i}^{ \pm}\left(m_{n}\right)=0, \quad a_{i j}<0, \quad 0 \leq i<j<N,
\end{gathered}
$$

where Sym denotes symmetrization with respect to the indices $\left(m_{1}, m_{2}\right)$. 
The generating functions are defined by

$$
x_{i}^{ \pm}(z)=\sum_{k \in \mathbb{Z}} x_{i}^{ \pm}(k) z^{-k}, \quad \omega_{i}(z)=\sum_{m \in \mathbb{Z}_{+}} \omega_{i}(m) z^{-m}, \quad \omega_{i}^{\prime}(z)=\sum_{n \in-\mathbb{Z}_{+}} \omega_{i}^{\prime}(n) z^{-n} .
$$

Remark 8.2. The subalgebra generated by $x_{i}^{ \pm}(k), a_{i}(m), \omega_{i}^{ \pm 1}, \omega_{i}^{\prime \pm 1}, \gamma^{ \pm \frac{1}{2}}, \gamma^{\prime \pm \frac{1}{2}}$, $D^{ \pm 1}, D^{\prime \pm 1}$ with indices $1 \leq i \leq N$ is a two-parameter quantum affine algebra of $A D E$ type (cf. $\mathrm{HZ}, \mathrm{Z}$ ).

In the case of type $A$, the two-parameter quantum toroidal algebra $U_{r, s}(\widehat{\widehat{\mathfrak{g}}})$ admits a further deformation $U_{r, s, \kappa}(\widehat{\mathfrak{g}})$. Let $\left(b_{i j}\right)$ be the skew-symmetric $(N+1) \times(N+1)-$ matrix

$$
\left(\begin{array}{cccccc}
0 & 1 & 0 & \ldots & 0 & -1 \\
-1 & 0 & 1 & \ldots & 0 & 0 \\
\cdots & \ldots & \ldots & \ldots & \ldots & \ldots \\
0 & 0 & 0 & \ldots & 0 & 1 \\
1 & 0 & 0 & \ldots & -1 & 0
\end{array}\right) .
$$

Definition 8.3. Let $\kappa$ be an element of $\mathbb{K}^{*}$. The two-parameter quantum toroidal algebra $U_{r, s, \kappa}(\widehat{\widehat{\mathfrak{g}}})$ is an associative algebra over $\mathbb{K}$ generated by the elements $x_{i}^{ \pm}(k)$, $a_{i}(m), \omega_{i}^{ \pm 1}, \omega_{i}^{\prime \pm 1}, \gamma^{ \pm \frac{1}{2}}, \gamma^{\prime \pm \frac{1}{2}}, D_{1}^{ \pm 1}, D_{1}^{\prime \pm 1}, D_{2}^{ \pm 1}, D_{2}^{\prime \pm 1}\left(0 \leq i \leq N, k, k^{\prime} \in \mathbb{Z}\right.$, $\left.m, m^{\prime} \in \mathbb{Z} \backslash\{0\}, l, l^{\prime}=1,2\right)$, subject to the following defining relations:

(T1) $\gamma^{ \pm \frac{1}{2}}, \gamma^{ \pm \frac{1}{2}}$ are central with $\gamma \gamma^{\prime}=r s, \omega_{i} \omega_{i}^{-1}=\omega_{j}^{\prime} \omega_{j}^{\prime-1}=1(i, j \in I)$, and

$$
\begin{aligned}
& {\left[\omega_{i}^{ \pm 1}, \omega_{j}^{ \pm 1}\right]=\left[\omega_{i}^{ \pm 1}, D_{l}^{ \pm 1}\right]=\left[\omega_{j}^{\prime \pm 1}, D_{l}^{ \pm 1}\right]=\left[\omega_{i}^{ \pm 1}, D_{l}^{\prime \pm 1}\right]=0} \\
& =\left[\omega_{i}^{ \pm 1}, \omega_{j}^{\prime \pm 1}\right]=\left[\omega_{j}^{\prime \pm 1}, D_{l}^{\prime \pm 1}\right]=\left[D_{l}^{\prime \pm 1}, D_{l^{\prime}}^{ \pm 1}\right]=\left[\omega_{i}^{\prime \pm 1}, \omega_{j}^{\prime \pm 1}\right] \text {. } \\
& {\left[a_{i}(m), a_{j}\left(m^{\prime}\right)\right]=\delta_{m+m^{\prime}, 0} \frac{(r s)^{\frac{|m|}{2}}\left(A_{i i}^{\frac{m a_{i j}}{2}}-A_{i i}^{-\frac{m a_{i j}}{2}}\right)}{|m|(r-s)} \cdot \frac{\gamma^{|m|}-\gamma^{\prime|m|}}{r-s} \kappa^{m b_{i j}} \text {. }} \\
& {\left[a_{i}(m), \omega_{j}^{ \pm 1}\right]=\left[a_{i}(m), \omega_{j}^{\prime \pm 1}\right]=0 .} \\
& D_{1} x_{i}^{ \pm}(k) D_{1}^{-1}=r^{k} x_{i}^{ \pm}(k), \quad D_{1}^{\prime} x_{i}^{ \pm}(k) D_{1}^{\prime-1}=s^{k} x_{i}^{ \pm}(k), \\
& D_{1} a_{i}(m) D_{1}^{-1}=r^{m} a_{i}(m), \quad D_{1}^{\prime} a_{i}(m) D_{1}^{\prime-1}=s^{m} a_{i}(m), \\
& D_{2} x_{i}^{ \pm}(k) D_{2}^{-1}=r^{ \pm \delta_{i 0}} x_{i}^{ \pm}(k), \quad D_{2}^{\prime} x_{i}^{ \pm}(k) D_{2}^{\prime-1}=s^{ \pm \delta_{i 0}} x_{i}^{ \pm}(k) \text {, } \\
& D_{2} a_{i}(m) D_{2}^{-1}=a_{i}(m), \quad D_{2}^{\prime} a_{i}(m) D_{2}^{\prime-1}=a_{i}(m) \text {. } \\
& \omega_{i}^{\prime} x_{j}^{ \pm}(k) \omega_{i}^{\prime-1}=A_{i j}^{\mp 1} x_{j}^{ \pm}(k) \text {. } \\
& {\left[a_{i}(m), x_{j}^{ \pm}(k)\right]= \pm \frac{(r s)^{\frac{|m|}{2}}\left(\left(r s^{-1}\right)^{\frac{m a_{i j}}{2}}-\left(r s^{-1}\right)^{-\frac{m a_{i j}}{2}}\right)}{m(r-s)}} \\
& \cdot \gamma^{ \pm \frac{m}{2}} \kappa^{m b_{i j}} x_{j}^{ \pm}(m+k), \quad \text { for } \quad m<0, \\
& {\left[a_{i}(m), x_{j}^{ \pm}(k)\right]= \pm \frac{(r s)^{\frac{|m|}{2}}\left(\left(r s^{-1}\right)^{\frac{m a_{i j}}{2}}-\left(r s^{-1}\right)^{-\frac{m a_{i j}}{2}}\right)}{m(r-s)}} \\
& \cdot \gamma^{\prime \pm \frac{m}{2}} \kappa^{m b_{i j}} x_{j}^{ \pm}(m+k), \quad \text { for } \quad m>0 . \\
& \left(\kappa^{b_{i j}} z-\left(A_{i j} A_{j i}\right)^{ \pm \frac{1}{2}} w\right) x_{i}^{ \pm}(z) x_{j}^{ \pm}(w) \\
& =\left(\kappa^{b_{i j}} A_{j i}^{ \pm 1} z-\left(A_{j i} A_{i j}^{-1}\right)^{ \pm \frac{1}{2}} w\right) x_{j}^{ \pm}(w) x_{i}^{ \pm}(z) .
\end{aligned}
$$




$$
\left[x_{i}^{+}(k), x_{j}^{-}\left(k^{\prime}\right)\right]=\frac{\delta_{i j}}{r-s}\left(\gamma^{\prime-k} \gamma^{-\frac{k+k^{\prime}}{2}} \omega_{i}\left(k+k^{\prime}\right)-\gamma^{k^{\prime}} \gamma^{\prime \frac{k+k^{\prime}}{2}} \omega_{i}^{\prime}\left(k+k^{\prime}\right)\right),
$$

where $\omega_{i}(m), \omega_{i}^{\prime}(-m)\left(m \in \mathbb{Z}_{\geq 0}\right)$ with $\omega_{i}(0)=\omega_{i}$ and $\omega_{i}^{\prime}(0)=\omega_{i}^{\prime}$ are defined by

$$
\begin{aligned}
\sum_{m=0}^{\infty} \omega_{i}(m) z^{-m} & =\omega_{i} \exp \left((r-s) \sum_{\ell=1}^{\infty} a_{i}(\ell) z^{-\ell}\right), \\
\sum_{m=0}^{\infty} \omega_{i}^{\prime}(-m) z^{m} & =\omega_{i}^{\prime} \exp \left(-(r-s) \sum_{\ell=1}^{\infty} a_{i}(-\ell) z^{\ell}\right),
\end{aligned}
$$

with $\omega_{i}(-m)=0$ and $\omega_{i}^{\prime}(m)=0, \forall m>0$.

$$
\begin{gathered}
\operatorname{Sym}_{m_{1}, \cdots, m_{n}} \sum_{k=0}^{n=1-a_{i j}}(-1)^{k}\left(r_{i} s_{i}\right)^{ \pm \frac{k(k-1)}{2}}\left[\begin{array}{c}
1-a_{i j} \\
k
\end{array}\right]_{ \pm i} x_{i}^{ \pm}\left(m_{1}\right) \cdots x_{i}^{ \pm}\left(m_{k}\right) x_{j}^{ \pm}(\ell) \\
\times x_{i}^{ \pm}\left(m_{k+1}\right) \cdots x_{i}^{ \pm}\left(m_{n}\right)=0, \quad a_{i j}<0, \quad 0 \leq j<i<N,
\end{gathered}
$$

$$
\begin{gathered}
\operatorname{Sym}_{m_{1}, \cdots, m_{n}} \sum_{k=0}^{n=1-a_{i j}}(-1)^{k}\left(r_{i} s_{i}\right)^{\mp \frac{k(k-1)}{2}}\left[\begin{array}{c}
1-a_{i j} \\
k
\end{array}\right]_{\mp i} x_{i}^{ \pm}\left(m_{1}\right) \cdots x_{i}^{ \pm}\left(m_{k}\right) x_{j}^{ \pm}(\ell) \\
\times x_{i}^{ \pm}\left(m_{k+1}\right) \cdots x_{i}^{ \pm}\left(m_{n}\right)=0, \quad a_{i j}<0, \quad 0 \leq i<j<N .
\end{gathered}
$$

Sym denotes symmetrization with respect to the indices $\left(m_{1}, m_{2}\right)$.

Remark 8.4. Assume that $r=s^{-1}=q$ and $U_{r, s, \kappa}(\widehat{\widehat{\mathfrak{g}}})$ is the one-parameter quantum toroidal algebra $U_{q, \kappa}(\widehat{\widehat{\mathfrak{g}}})$ (cf. GKV, $\left.\mathrm{VV}\right]$ ).

8.2. A new form of McKay correspondence. In this subsection we let $\Gamma$ be a finite subgroup of $S U_{2}$ and consider two distinguished choices of the class function $\xi$ in $R_{\Gamma \times \mathbb{C} \times \times \mathbb{C} \times}$ introduced in Section 4.2 .

First we consider

$$
\xi=\gamma_{0} \otimes\left(\left(r s^{-1}\right)^{\frac{1}{2}}+\left(r^{-1} s\right)^{\frac{1}{2}}\right)-\pi \otimes 1_{\mathbb{C}^{\times}},
$$

where $\pi$ is the character of the two-dimensional natural representation of $\Gamma$ in $S U_{2}$.

The Heisenberg algebra in this case has the following relations (cf. Proposition 5.1 and (4.2) :

$$
\left[a_{m}\left(\gamma_{i}\right), a_{n}\left(\gamma_{j}\right)\right]= \begin{cases}m \delta_{m,-n}\left(\left(r s^{-1}\right)^{\frac{m}{2}}+\left(r^{-1} s\right)^{\frac{m}{2}}\right) C, & i=j, \\ m \delta_{m,-n} a_{i j}^{1} C, & i \neq j,\end{cases}
$$

where $a_{i j}^{1}$ are the entries of the affine Cartan matrix of ADE type (see (3.4) at $d=2)$.

Recall that the matrix $A^{1,1}=\left(\left\langle\gamma_{i}, \gamma_{j}\right\rangle_{\xi}^{1}\right)=\left(a_{i j}^{1}\right)_{0 \leq i, j \leq N}$ is the Cartan matrix for the corresponding affine Lie algebra [Mc]. In particular $a_{i i}^{1}=2, a_{i j}^{1}=0$ or -1 when $i \neq j$ and $\Gamma \neq \mathbb{Z} / 2 \mathbb{Z}$. In the case of $\Gamma=\mathbb{Z} / 2 \mathbb{Z}, a_{01}^{1}=a_{10}^{1}=-2$. Let $\mathfrak{g}$ (resp. $\hat{\mathfrak{g}}$ ) be the corresponding simple Lie algebra (resp. affine Lie algebra ) associated to the Cartan matrix $\left(a_{i j}^{1}\right)_{1 \leq i, j \leq N}$ (resp. $A$ ). Note that the lattice $R_{\mathbb{Z}}(\Gamma)$ is even in this case. 
We define the normal ordered product of vertex operators as follows:

$$
\begin{aligned}
& : Y^{+}\left(\gamma_{i}, a, b, z\right) Y^{+}\left(\gamma_{j}, a^{\prime}, b^{\prime}, w\right): \\
= & H_{+}\left(\gamma_{i}, z\right) H\left(\gamma_{j}, w\right) S\left(H_{+}\left(\gamma_{i} \otimes r^{a} s^{b}, z^{-1}\right)^{*} H_{+}\left(\gamma_{j} \otimes r^{a^{\prime}} s^{b^{\prime}}, w^{-1}\right)^{*}\right) \\
& \times e^{\gamma_{i}+\gamma_{j}} z^{\partial_{\gamma_{i}}} w^{\partial_{\gamma_{j}}} \\
& : Y^{+}\left(\gamma_{i}, a, b, z\right) Y^{-}\left(\gamma_{j}, a^{\prime}, b^{\prime}, w\right): \\
= & H_{+}\left(\gamma_{i}, z\right) H\left(-\gamma_{j} \otimes r^{b^{\prime}} s^{a^{\prime}}, w\right) S\left(H_{+}\left(\gamma_{i} \otimes r^{a} s^{b}, z^{-1}\right)^{*} H_{+}\left(-\gamma_{j} \otimes r^{a^{\prime}} s^{b^{\prime}}, w^{-1}\right)^{*}\right) \\
& \times e^{\gamma_{i}-\gamma_{j}} z^{\partial_{\gamma_{i}}} w^{-\partial_{\gamma_{j}}} .
\end{aligned}
$$

Other normal ordered products are defined similarly.

The identities in the following theorems are understood as usual by means of correlation functions (cf. e.g. [FJ, J1, HZ, Z]).

Theorem 8.5. Let $\xi=\gamma_{0} \otimes\left(\left(r s^{-1}\right)^{\frac{1}{2}}+\left(r^{-1} s\right)^{\frac{1}{2}}\right)-\pi \otimes 1_{\mathbb{C}^{\times}}$. Then the vertex operators $Y^{ \pm}\left(\gamma_{i}, a, b, z\right), Y^{ \pm}\left(-\gamma_{j}, a, b, z\right), \gamma_{i} \in \Gamma^{*}, a, b \in \mathbb{Z}$ acting on the group-theoretically defined Fock space $\mathcal{F}_{\Gamma \times \mathbb{C}^{\times} \times \mathbb{C}^{\times}}$satisfy the following relations:

$$
\begin{aligned}
& Y^{+}\left(\gamma_{i}, a, b, s^{-b} z\right) Y^{+}\left(\gamma_{j}, a, b, s^{-b} w\right) \\
& =\epsilon\left(\gamma_{i}, \gamma_{j}\right): Y^{+}\left(\gamma_{i}, a, b, s^{-b} z\right) Y^{+}\left(\gamma_{j}, a, b, s^{-b} w\right): \\
& \times\left\{\begin{array}{cc}
1 & \left\langle\gamma_{i}, \gamma_{j}\right\rangle_{\xi}^{1}=0, \\
\left(z-r^{-a} s^{-b} w\right)^{-1} & \left\langle\gamma_{i}, \gamma_{j}\right\rangle_{\xi}^{1}=-1, \\
\left(z-r^{-a} s^{-b}\left(r s^{-1}\right)^{\frac{1}{2}} w\right)\left(z-r^{-a} s^{-b}\left(r^{-1} s\right)^{\frac{1}{2}} w\right) & \left\langle\gamma_{i}, \gamma_{j}\right\rangle_{\xi}^{1}=2,
\end{array}\right. \\
& Y^{-}\left(\gamma_{i}, a, b, r^{-a} z\right) Y^{-}\left(\gamma_{j}, a, b, r^{-a} w\right) \\
& =\epsilon\left(\gamma_{i}, \gamma_{j}\right)^{-1}: Y^{-}\left(\gamma_{i}, a, b, r^{-a} z\right) Y^{-}\left(\gamma_{j}, a, b, r^{-a} w\right): \\
& \times\left\{\begin{array}{cc}
1 & \left\langle\gamma_{i}, \gamma_{j}\right\rangle_{\xi}^{1}=0, \\
\left(z-r^{a} s^{b} w\right)^{-1} & \left\langle\gamma_{i}, \gamma_{j}\right\rangle_{\xi}^{1}=-1, \\
\left(z-r^{a} s^{b}\left(r s^{-1}\right)^{\frac{1}{2}} w\right)\left(z-r^{a} s^{b}\left(r^{-1} s\right)^{\frac{1}{2}} w\right) & \left\langle\gamma_{i}, \gamma_{j}\right\rangle_{\xi}^{1}=2,
\end{array}\right. \\
& Y^{+}\left(\gamma_{i}, a, b, s^{-b} z\right) Y^{-}\left(\gamma_{j}, a, b, r^{-a} w\right) \\
& =\epsilon\left(\gamma_{i}, \gamma_{j}\right): Y^{+}\left(\gamma_{i}, a, b, s^{-b} z\right) Y^{-}\left(\gamma_{j}, a, b, r^{-a} w\right): \\
& \times\left\{\begin{array}{cc}
1 & \left\langle\gamma_{i}, \gamma_{j}\right\rangle_{\xi}^{1}=0, \\
\left(z-r^{-a} s^{b} w\right) & \left\langle\gamma_{i}, \gamma_{j}\right\rangle_{\xi}^{1}=-1, \\
\left(z-r^{-a} s^{b}\left(r s^{-1}\right)^{\frac{1}{2}} w\right)\left(z-r^{-a} s^{b}\left(r^{-1} s\right)^{\frac{1}{2}} w\right) & \left\langle\gamma_{i}, \gamma_{j}\right\rangle_{\xi}^{1}=2,
\end{array}\right. \\
& Y^{-}\left(\gamma_{i}, a, b, r^{-a} z\right) Y^{+}\left(\gamma_{j}, a, b, s^{-b} w\right) \\
& =\epsilon\left(\gamma_{i}, \gamma_{j}\right)^{-1}: Y^{-}\left(\gamma_{i}, a, b, r^{-a} z\right) Y^{+}\left(\gamma_{j}, a, b, s^{-b} w\right): \\
& \times\left\{\begin{array}{cc}
1 & \left\langle\gamma_{i}, \gamma_{j}\right\rangle_{\xi}^{1}=0, \\
\left(z-r^{a} s^{-b} w\right) & \left\langle\gamma_{i}, \gamma_{j}\right\rangle_{\xi}^{1}=-1, \\
\left(z-r^{a} s^{-b}\left(r s^{-1}\right)^{\frac{1}{2}} w\right)\left(z-r^{a} s^{-b}\left(r^{-1} s\right)^{\frac{1}{2}} w\right) & \left\langle\gamma_{i}, \gamma_{j}\right\rangle_{\xi}^{1}=2,
\end{array}\right. \\
& Y^{+}\left(-\gamma_{i},-a,-b, s^{-b} z\right) Y^{+}\left(-\gamma_{j},-a,-b, s^{-b} w\right) \\
& =\epsilon\left(\gamma_{i}, \gamma_{j}\right): Y^{+}\left(-\gamma_{i},-a,-b, s^{-b} z\right) Y^{+}\left(-\gamma_{j},-a,-b, s^{-b} w\right): \\
& \times\left\{\begin{array}{cc}
1 & \left\langle\gamma_{i}, \gamma_{j}\right\rangle_{\xi}^{1}=0, \\
\left(z-r^{-a} s^{-b} w\right)^{-1} & \left\langle\gamma_{i}, \gamma_{j}\right\rangle_{\xi}^{1}=-1, \\
\left(z-r^{-a} s^{-b}\left(r s^{-1}\right)^{\frac{1}{2}} w\right)\left(z-r^{-a} s^{-b}\left(r^{-1} s\right)^{\frac{1}{2}} w\right) & \left\langle\gamma_{i}, \gamma_{j}\right\rangle_{\xi}^{1}=2
\end{array}\right.
\end{aligned}
$$




$$
\begin{aligned}
& Y^{-}\left(-\gamma_{i},-a,-b, r^{-a} z\right) Y^{+}\left(-\gamma_{j},-a,-b, r^{-a} w\right) \\
& =\epsilon\left(\gamma_{i}, \gamma_{j}\right)^{-1}: Y^{-}\left(-\gamma_{i},-a,-b, r^{-a} z\right) Y^{+}\left(-\gamma_{j},-a,-b, r^{-a} w\right) \text { : } \\
& \times\left\{\begin{array}{cc}
1 & \left\langle\gamma_{i}, \gamma_{j}\right\rangle_{\xi}^{1}=0, \\
\left(z-r^{a} s^{b} w\right)^{-1} & \left\langle\gamma_{i}, \gamma_{j}\right\rangle_{\xi}^{1}=-1, \\
\left(z-r^{a} s^{b}\left(r s^{-1}\right)^{\frac{1}{2}} w\right)\left(z-r^{a} s^{b}\left(r^{-1} s\right)^{\frac{1}{2}} w\right) & \left\langle\gamma_{i}, \gamma_{j}\right\rangle_{\xi}^{1}=2,
\end{array}\right. \\
& Y^{+}\left(-\gamma_{i},-a,-b, s^{-b} z\right) Y^{-}\left(-\gamma_{j},-a,-b, r^{-a} w\right) \\
& =\epsilon\left(\gamma_{i}, \gamma_{j}\right): Y^{+}\left(-\gamma_{i},-a,-b, s^{-b} z\right) Y^{-}\left(-\gamma_{j},-a,-b, r^{-a} w\right): \\
& \times\left\{\begin{array}{cc}
1 & \left\langle\gamma_{i}, \gamma_{j}\right\rangle_{\xi}^{1}=0, \\
\left(z-r^{-a} s^{b} w\right) & \left\langle\gamma_{i}, \gamma_{j}\right\rangle_{\xi}^{1}=-1, \\
\left(z-r^{-a} s^{b}\left(r s^{-1}\right)^{\frac{1}{2}} w\right)\left(z-r^{-a} s^{b}\left(r^{-1} s\right)^{\frac{1}{2}} w\right) & \left\langle\gamma_{i}, \gamma_{j}\right\rangle_{\xi}^{1}=2,
\end{array}\right. \\
& Y^{-}\left(-\gamma_{i},-a,-b, r^{-a} z\right) Y^{+}\left(-\gamma_{j},-a,-b, s^{-b} w\right) \\
& =\epsilon\left(\gamma_{i}, \gamma_{j}\right)^{-1}: Y^{-}\left(-\gamma_{i},-a,-b, r^{-a} z\right) Y^{+}\left(-\gamma_{j},-a,-b, s^{-b} w\right): \\
& \times\left\{\begin{array}{cc}
1 & \left\langle\gamma_{i}, \gamma_{j}\right\rangle_{\xi}^{1}=0, \\
\left(z-r^{a} s^{-b} w\right) & \left\langle\gamma_{i}, \gamma_{j}\right\rangle_{\xi}^{1}=-1, \\
\left(z-r^{a} s^{-b}\left(r s^{-1}\right)^{\frac{1}{2}} w\right)\left(z-r^{a} s^{-b}\left(r^{-1} s\right)^{\frac{1}{2}} w\right) & \left\langle\gamma_{i}, \gamma_{j}\right\rangle_{\xi}^{1}=2 .
\end{array}\right.
\end{aligned}
$$

Remark 8.6. Replacing the vertex operator $Y^{ \pm}$by $X^{ \pm}$via the characteristic map $c h$ in the above formulas, we get the corresponding formulas for vertex operators $X^{ \pm}(\gamma, a, b, z)$ acting on $V_{\Gamma \times \mathbb{C}^{\times} \times \mathbb{C}^{\times}}$.

Now we consider the second distinguished class function

$$
\xi^{r, s, \kappa}=\gamma_{0} \otimes\left(\left(r s^{-1}\right)^{\frac{1}{2}}+\left(r^{-1} s\right)^{\frac{1}{2}}\right)-\left(\gamma_{1} \otimes \kappa+\gamma_{r} \otimes \kappa^{-1}\right),
$$

when $\Gamma$ is a cyclic group of order $N+1$.

In this case the Heisenberg algebra (5.3) has the following relations according to Proposition 5.1:

$$
\left[a_{m}\left(\gamma_{i}\right), a_{n}\left(\gamma_{j}\right)\right]= \begin{cases}m \delta_{m,-n}\left(\left(r s^{-1}\right)^{\frac{m}{2}}+\left(r^{-1} s\right)^{\frac{m}{2}}\right) \kappa^{m b_{i j}} C, & i=j, \\ m \delta_{m,-n} a_{i j}^{1} \kappa^{m b_{i j}} C, & i \neq j,\end{cases}
$$

where $a_{i j}^{1}$ are the entries of the affine Cartan matrix of type A and $r \geq 2$. This is the same Heisenberg subalgebra $(c=1)$ in $U_{r, s}(\widehat{\mathfrak{g}})$ provided that we identify

$$
a_{i}(n)=\frac{[n]}{n} a_{n}\left(\gamma_{i}\right)
$$

Recall that $\left(b_{i j}\right)$ is the skew-symmetric matrix. We need to slightly modify the definition of the middle term in the vertex operators. For each $i=0,1, \ldots, N$ we define the modified operator $z^{\partial_{\gamma, \kappa}}$ on the group algebra $\mathbb{C}\left[R_{\mathbb{Z}}(\Gamma)\right]$ by

$$
z^{\partial_{\gamma_{i}, \kappa}} e^{\beta}=z^{\left\langle\gamma_{i}, \beta\right\rangle_{\xi}^{1}} \kappa^{-\frac{1}{2} \sum_{j=1}^{r}\left\langle\gamma_{i}, m_{j} \gamma_{j}\right\rangle_{\xi}^{1} b_{i j}} e^{\beta},
$$

where $\beta=\sum_{j} m_{j} \gamma_{j} \in R_{\mathbb{Z}}(\Gamma)$.

We then replace the operator $z^{ \pm \partial_{\gamma_{i}}}$ in the definition of the vertex operators $Y^{ \pm}\left(\gamma_{i}, a, b, z\right)$ by the operator $z^{ \pm \partial_{\gamma_{i}, \kappa}}$. The formulas in Theorem 7.4 remain true after the terms $z^{ \pm \partial}$ appearing in the formulas are modified accordingly.

The proof of the following theorem is similar to that of Theorem 8.5 
Theorem 8.7. Let $\Gamma$ be a cyclic group of order $r+1$ and let $\xi^{r, s, \kappa}=\gamma_{0} \otimes$ $\left(\left(r s^{-1}\right)^{\frac{1}{2}}+\left(r^{-1} s\right)^{\frac{1}{2}}\right)-\left(\gamma_{1} \otimes \kappa+\gamma_{r} \otimes \kappa^{-1}\right)$. The vertex operators $Y^{ \pm}\left(\gamma_{i}, a, b, z\right)$ and $Y^{ \pm}\left(-\gamma_{i}, a, b, z\right), \gamma_{i} \in \Gamma^{*}$ acting on the group-theoretically defined Fock space $\mathcal{F}_{\Gamma \times \mathbb{C} \times \times \mathbb{C} \times}$ satisfy the following relations:

$$
\begin{aligned}
& Y^{+}\left(\gamma_{i}, a, b, s^{-b} z\right) Y^{+}\left(\gamma_{j}, a, b, s^{-b} w\right) \\
& =\epsilon\left(\gamma_{i}, \gamma_{j}\right): Y^{+}\left(\gamma_{i}, a, b, s^{-b} z\right) Y^{+}\left(\gamma_{j}, a, b, s^{-b} w\right): \\
& \times\left\{\begin{array}{cc}
1 & \left\langle\gamma_{i}, \gamma_{j}\right\rangle_{\xi}^{1}=0, \\
\kappa^{-\frac{1}{2} b_{i j}}\left(z-r^{-a} s^{-b} \kappa^{b_{i j}} w\right)^{-1} & \left\langle\gamma_{i}, \gamma_{j}\right\rangle_{\xi}^{1}=-1, \\
\left(z-r^{-a} s^{-b}\left(r s^{-1}\right)^{\frac{1}{2}} w\right)\left(z-r^{-a} s^{-b}\left(r^{-1} s\right)^{\frac{1}{2}} w\right) & \left\langle\gamma_{i}, \gamma_{j}\right\rangle_{\xi}^{1}=2,
\end{array}\right. \\
& Y^{-}\left(\gamma_{i}, a, b, r^{-a} z\right) Y^{-}\left(\gamma_{j}, a, b, r^{-a} w\right) \\
& =\epsilon\left(\gamma_{i}, \gamma_{j}\right)^{-1}: Y^{-}\left(\gamma_{i}, a, b, r^{-a} z\right) Y^{-}\left(\gamma_{j}, a, b, r^{-a} w\right): \\
& \times\left\{\begin{array}{cc}
1 & \left\langle\gamma_{i}, \gamma_{j}\right\rangle_{\xi}^{1}=0, \\
\kappa^{-\frac{1}{2} b_{i j}}\left(z-r^{a} s^{b} \kappa^{b_{i j}} w\right)^{-1} & \left\langle\gamma_{i}, \gamma_{j}\right\rangle_{\xi}^{1}=-1, \\
\left(z-r^{a} s^{b}\left(r s^{-1}\right)^{\frac{1}{2}} w\right)\left(z-r^{a} s^{b}\left(r^{-1} s\right)^{\frac{1}{2}} w\right) & \left\langle\gamma_{i}, \gamma_{j}\right\rangle_{\xi}^{1}=2,
\end{array}\right. \\
& Y^{+}\left(\gamma_{i}, a, b, s^{-b} z\right) Y^{-}\left(\gamma_{j}, a, b, r^{-a} w\right) \\
& =\epsilon\left(\gamma_{i}, \gamma_{j}\right): Y^{+}\left(\gamma_{i}, a, b, s^{-b} z\right) Y^{-}\left(\gamma_{j}, a, b, r^{-a} w\right): \\
& \times\left\{\begin{array}{cc}
1 & \left\langle\gamma_{i}, \gamma_{j}\right\rangle_{\xi}^{1}=0, \\
\kappa^{-\frac{1}{2} b_{i j}}\left(z-r^{-a} s^{b} \kappa^{b_{i j}} w\right) & \left\langle\gamma_{i}, \gamma_{j}\right\rangle_{\xi}^{1}=-1, \\
\left(z-r^{-a} s^{b}\left(r s^{-1}\right)^{\frac{1}{2}} w\right)\left(z-r^{-a} s^{b}\left(r^{-1} s\right)^{\frac{1}{2}} w\right) & \left\langle\gamma_{i}, \gamma_{j}\right\rangle_{\xi}^{1}=2,
\end{array}\right. \\
& Y^{-}\left(\gamma_{i}, a, b, r^{-a} z\right) Y^{+}\left(\gamma_{j}, a, b, s^{-b} w\right) \\
& =\epsilon\left(\gamma_{i}, \gamma_{j}\right)^{-1}: Y^{-}\left(\gamma_{i}, a, b, r^{-a} z\right) Y^{+}\left(\gamma_{j}, a, b, s^{-b} w\right) \text { : } \\
& \times\left\{\begin{array}{cc}
1 & \left\langle\gamma_{i}, \gamma_{j}\right\rangle_{\xi}^{1}=0, \\
\kappa^{-\frac{1}{2} b_{i j}}\left(z-r^{a} s^{-b} \kappa^{b_{i j}} w\right) & \left\langle\gamma_{i}, \gamma_{j}\right\rangle_{\xi}^{1}=-1, \\
\left(z-r^{a} s^{-b}\left(r s^{-1}\right)^{\frac{1}{2}} w\right)\left(z-r^{a} s^{-b}\left(r^{-1} s\right)^{\frac{1}{2}} w\right) & \left\langle\gamma_{i}, \gamma_{j}\right\rangle_{\xi}^{1}=2,
\end{array}\right. \\
& Y^{+}\left(-\gamma_{i},-a,-b, s^{-b} z\right) Y^{+}\left(-\gamma_{j},-a,-b, s^{-b} w\right) \\
& =\epsilon\left(\gamma_{i}, \gamma_{j}\right): Y^{+}\left(-\gamma_{i},-a,-b, s^{-b} z\right) Y^{+}\left(-\gamma_{j},-a,-b, s^{-b} w\right): \\
& \times\left\{\begin{array}{cc}
1 & \left\langle\gamma_{i}, \gamma_{j}\right\rangle_{\xi}^{1}=0, \\
\kappa^{-\frac{1}{2} b_{i j}}\left(z-r^{-a} s^{-b} \kappa^{b_{i j}} w\right)^{-1} & \left\langle\gamma_{i}, \gamma_{j}\right\rangle_{\xi}^{1}=-1, \\
\left(z-r^{-a} s^{-b}\left(r s^{-1}\right)^{\frac{1}{2}} w\right)\left(z-r^{-a} s^{-b}\left(r^{-1} s\right)^{\frac{1}{2}} w\right) & \left\langle\gamma_{i}, \gamma_{j}\right\rangle_{\xi}^{1}=2,
\end{array}\right. \\
& Y^{-}\left(-\gamma_{i},-a,-b, r^{-a} z\right) Y^{+}\left(-\gamma_{j},-a,-b, r^{-a} w\right) \\
& =\epsilon\left(\gamma_{i}, \gamma_{j}\right)^{-1}: Y^{-}\left(-\gamma_{i},-a,-b, r^{-a} z\right) Y^{+}\left(-\gamma_{j},-a,-b, r^{-a} w\right): \\
& \times\left\{\begin{array}{cc}
1 & \left\langle\gamma_{i}, \gamma_{j}\right\rangle_{\xi}^{1}=0, \\
\kappa^{-\frac{1}{2} b_{i j}}\left(z-r^{a} s^{b} \kappa^{b_{i j}} w\right)^{-1} & \left\langle\gamma_{i}, \gamma_{j}\right\rangle_{\xi}^{1}=-1, \\
\left(z-r^{a} s^{b}\left(r s^{-1}\right)^{\frac{1}{2}} w\right)\left(z-r^{a} s^{b}\left(r^{-1} s\right)^{\frac{1}{2}} w\right) & \left\langle\gamma_{i}, \gamma_{j}\right\rangle_{\xi}^{1}=2,
\end{array}\right. \\
& Y^{+}\left(-\gamma_{i},-a,-b, s^{-b} z\right) Y^{-}\left(-\gamma_{j},-a,-b, r^{-a} w\right) \\
& =\epsilon\left(\gamma_{i}, \gamma_{j}\right): Y^{+}\left(-\gamma_{i},-a,-b, s^{-b} z\right) Y^{-}\left(-\gamma_{j},-a,-b, r^{-a} w\right) \text { : } \\
& \times\left\{\begin{array}{cc}
1 & \left\langle\gamma_{i}, \gamma_{j}\right\rangle_{\xi}^{1}=0 \\
\kappa^{-\frac{1}{2} b_{i j}}\left(z-r^{-a} s^{b} \kappa^{b_{i j}} w\right) & \left\langle\gamma_{i}, \gamma_{j}\right\rangle_{\xi}^{1}=-1, \\
\left(z-r^{-a} s^{b}\left(r s^{-1}\right)^{\frac{1}{2}} w\right)\left(z-r^{-a} s^{b}\left(r^{-1} s\right)^{\frac{1}{2}} w\right) & \left\langle\gamma_{i}, \gamma_{j}\right\rangle_{\xi}^{1}=2,
\end{array}\right.
\end{aligned}
$$




$$
\begin{aligned}
& Y^{-}\left(-\gamma_{i},-a,-b, r^{-a} z\right) Y^{+}\left(-\gamma_{j},-a,-b, s^{-b} w\right) \\
& \quad=\epsilon\left(\gamma_{i}, \gamma_{j}\right)^{-1}: Y^{-}\left(-\gamma_{i},-a,-b, r^{-a} z\right) Y^{+}\left(-\gamma_{j},-a,-b, s^{-b} w\right): \\
& \quad \times\left\{\begin{array}{cc}
1 & \left\langle\gamma_{i}, \gamma_{j}\right\rangle_{\xi}^{1}=0, \\
\kappa^{-\frac{1}{2} b_{i j}}\left(z-r^{a} s^{-b} \kappa^{b_{i j}} w\right) & \left\langle\gamma_{i}, \gamma_{j}\right\rangle_{\xi}^{1}=-1, \\
\left(z-r^{a} s^{-b}\left(r s^{-1}\right)^{\frac{1}{2}} w\right)\left(z-r^{a} s^{-b}\left(r^{-1} s\right)^{\frac{1}{2}} w\right) & \left\langle\gamma_{i}, \gamma_{j}\right\rangle_{\xi}^{1}=2 .
\end{array}\right.
\end{aligned}
$$

Remark 8.8. Replacing the vertex operators $Y^{ \pm}$by $X^{ \pm}$via the characteristic map $c h$ we obtain the corresponding results on the space $V_{\Gamma \times \mathbb{C}^{\times} \times \mathbb{C}^{\times}}$.

8.3. Quantum vertex representations of $U_{r, s}(\widehat{\widehat{g}})$. For each $i=0, \ldots, N$ let

$$
\widetilde{a_{i}}(n)=\frac{[n]}{n} a_{n}\left(\gamma_{i}\right)
$$

It follows from (5.3) and (8.1) that

$$
\left[\widetilde{a_{i}}(m), \widetilde{a_{j}}(n)\right]=\delta_{m,-n} \frac{(r s)^{\frac{m\left(1-a_{i j}\right)}{2}}\left[m\left\langle\gamma_{i}, \gamma_{j}\right\rangle_{\xi}^{1}\right]}{m}[m] .
$$

According to McKay, the bilinear form $\left\langle\gamma_{i}, \gamma_{j}\right\rangle_{\xi}^{1}$ is exactly the same as the invariant form $(\mid)$ of the root lattice of the affine Lie algebra $\widehat{\mathfrak{g}}$. This implies that the commutation relations (8.4) are exactly the commutation relations (D2) of the Heisenberg algebra in $U_{r, s}(\widehat{\widehat{g}})$ if we identify $\widetilde{a_{i}}(n)$ with $a_{i}(n)$. Thus the Fock space $S_{\Gamma \times \mathbb{C}^{\times} \times \mathbb{C}^{\times}}$is a level one representation for the Heisenberg subalgebra in $U_{r, s}(\widehat{\widehat{g}})$.

The following theorem gives an $r, s$-deformation of the new form of McKay correspondences in FJW2 and provides a direct connection from a finite subgroup $\Gamma$ of $S U_{2}$ to the quantum toroidal algebra $U_{r, s}(\widehat{\widehat{g}})$ of $A D E$ type.

Theorem 8.9. Given a finite subgroup $\Gamma$ of $S_{2}$, each of the following correspondences gives a vertex representation of the quantum toroidal algebra $U_{r, s}(\widehat{\widehat{g}})$ on the Fock space $\mathcal{F}_{\Gamma \times \mathbb{C}^{\times} \times \mathbb{C}^{\times}}$:

$$
\begin{aligned}
x_{i}^{+}(n) & \longrightarrow Y_{n}^{+}\left(\gamma_{i} \otimes s^{b}, \frac{1}{2},-\frac{1}{2}\right), \\
x_{i}^{-}(n) & \longrightarrow Y_{n}^{-}\left(\gamma_{i} \otimes r^{a}, \frac{1}{2},-\frac{1}{2}\right), \\
a_{i}(m) & \longrightarrow \frac{[m]}{m} a_{m}\left(\gamma_{i}\right), \quad \text { for } \quad m>0, \\
a_{i}(m) & \longrightarrow \frac{-[-m]}{m} a_{m}\left(\gamma_{i}\right), \quad \text { for } \quad m<0, \\
\gamma & \longrightarrow r, \quad \gamma^{\prime} \longrightarrow s,
\end{aligned}
$$

or

$$
\begin{aligned}
x_{i}^{+}(n) & \longrightarrow Y_{n}^{-}\left(-\gamma_{i} \otimes s^{b},-\frac{1}{2}, \frac{1}{2}\right), \\
x_{i}^{-}(n) & \longrightarrow Y_{n}^{+}\left(-\gamma_{i} \otimes r^{a},-\frac{1}{2}, \frac{1}{2}\right), \\
a_{i}(m) & \longrightarrow \frac{[m]}{m} a_{m}\left(\gamma_{i}\right), \quad \text { for } m>0, \\
a_{i}(m) & \longrightarrow \frac{-[-m]}{m} a_{m}\left(\gamma_{i}\right), \quad \text { for } m<0, \\
\gamma & \longrightarrow r, \quad \gamma^{\prime} \longrightarrow s,
\end{aligned}
$$

where $i=0, \ldots, N$, and $m \in \mathbb{Z}$. 
Remark 8.10. Replacing $Y^{ \pm}$by $X^{ \pm}$in the above theorem, we obtain a vertex representation of $U_{r, s}(\widehat{\widehat{g}})$ in the space $V_{\Gamma \times \mathbb{C}^{\times} \times \mathbb{C}^{\times}}$.

According to McKay, the bilinear form $\left\langle\gamma_{i}, \gamma_{j}\right\rangle_{\xi}^{1}$ is exactly the same as the invariant form $(\mid)$ of the root lattice of the affine Lie algebra $\widehat{\mathfrak{g}}$. Thus the Fock space $S_{\Gamma \times \mathbb{C}^{\times} \times \mathbb{C}^{\times}}$is a level one representation for the Heisenberg subalgebra in $\mathcal{U}_{r, s}\left(\widehat{\mathfrak{s l}}_{n}\right)$.

Denote by $\bar{S}_{\Gamma \times \mathbb{C} \times}$ the symmetric algebra generated by $a_{-n}\left(\gamma_{i}\right), n>0, i=$ $1, \ldots, N$ over $\mathbb{C}\left[r^{ \pm 1}, s^{ \pm 1}\right] . \bar{S}_{\Gamma \times \mathbb{C}^{\times}}$is isometric to $\bar{R}_{\Gamma \times \mathbb{C}^{\times} \times \mathbb{C}^{\times}}$.

We define

$$
\overline{\mathcal{F}}_{\Gamma \times \mathbb{C}^{\times} \times \mathbb{C}^{\times}}=\bar{R}_{\Gamma \times \mathbb{C}^{\times} \times \mathbb{C}^{\times}} \otimes \mathbb{C}\left[\bar{R}_{\mathbb{Z}}(\Gamma)\right] \cong \bar{S}_{\Gamma \times \mathbb{C} \times} \otimes \mathbb{C}\left[\bar{R}_{\mathbb{Z}}(\Gamma)\right] .
$$

The space $V_{\Gamma \times \mathbb{C}^{\times} \times \mathbb{C}^{\times}}$associated to the lattice $R_{\mathbb{Z}}(\Gamma)$ is isomorphic to the tensor product of the space $\bar{R}_{\Gamma \times \mathbb{C}^{\times} \times \mathbb{C}^{\times}}$and $R_{\mathbb{Z}}(\Gamma)$ as well as the space associated to the rank 1 lattice $\mathbb{Z} \alpha_{0}$.

The following theorem gives the new form of McKay correspondence in FJW2 for the two-parameter case and provides a direct connection from a finite subgroup $\Gamma$ of $S U_{2}$ to the two-parameter quantum affine algebra $\mathcal{U}_{r, s}\left(\widehat{\mathfrak{s l}_{n}}\right)$.

Theorem 8.11. Given a finite subgroup $\Gamma$ of $\mathrm{SU}_{2}$, each of the following correspondences gives the basic representation of the two-parameter quantum affine algebra $\mathcal{U}_{r, s}\left(\widehat{\mathfrak{s l}}_{n}\right)$ on the Fock space $\overline{\mathcal{F}}_{\Gamma \times \mathbb{C} \times \times \mathbb{C} \times}$ :

$$
\begin{aligned}
x_{i}^{+}(n) & \longrightarrow Y_{n}^{+}\left(\gamma_{i} \otimes s^{b}, \frac{1}{2},-\frac{1}{2}\right), \\
x_{i}^{-}(n) & \longrightarrow Y_{n}^{-}\left(\gamma_{i} \otimes r^{a}, \frac{1}{2},-\frac{1}{2}\right), \\
a_{i}(m) & \longrightarrow \frac{[m]}{m} a_{m}\left(\gamma_{i}\right), \quad \text { for } m>0, \\
a_{i}(m) & \longrightarrow \frac{-[-m]}{m} a_{m}\left(\gamma_{i}\right), \quad \text { for } m<0, \\
\gamma & \longrightarrow r, \quad \gamma^{\prime} \longrightarrow s,
\end{aligned}
$$

or

$$
\begin{aligned}
x_{i}^{+}(n) & \longrightarrow Y_{n}^{-}\left(-\gamma_{i} \otimes s^{b},-\frac{1}{2}, \frac{1}{2}\right), \\
x_{i}^{-}(n) & \longrightarrow Y_{n}^{+}\left(-\gamma_{i} \otimes r^{a},-\frac{1}{2}, \frac{1}{2}\right), \\
a_{i}(m) & \longrightarrow \frac{[m]}{m} a_{m}\left(\gamma_{i}\right), \quad \text { for } m>0, \\
a_{i}(m) & \longrightarrow \frac{-[-m]}{m} a_{m}\left(\gamma_{i}\right), \quad \text { for } m<0, \\
\gamma & \longrightarrow r, \quad \gamma^{\prime} \longrightarrow s,
\end{aligned}
$$

where $i=1, \ldots, N$.

Remark 8.12. Replacing $Y^{ \pm}$by $X^{ \pm}$in the above theorem, we obtain a vertex representation of $\mathcal{U}_{r, s}(\widehat{\mathfrak{s l}})$ in the space $V_{\Gamma \times \mathbb{C}^{\times} \times \mathbb{C}^{\times}}(\mathrm{cf}$. [HZ, , Z] $)$, which generalizes the one-parameter cases [FJ, $\mathrm{S}$. 


\section{ACKNOWLEDGMENT}

The first author would like to thank the support of NSA grant and NSFC Overseas Collaborative Grant (No. 10728102). The second author would like to thank the support of NSFC (No. 10801094) and the Shanghai Leading Academic Discipline Project (No. J50101).

\section{REFERENCES}

[A] E. Abe, Hopf algebras, Cambridge Tracts in Mathematics, 74, Cambridge University Press, 1980. MR594432 (83a:16010)

[BGH1] N. Bergeron, Y. Gao, and N. Hu, Drinfel'd doubles and Lusztig's symmetries of twoparameter quantum groups, J. Algebra 301 (2006), 378-405. MR2230338 (2007e:17010)

[BGH2] N. Bergeron, Y. Gao, and N. Hu, Representations of two-parameter quantum orthogonal and symplectic groups, AMS/IP Studies in Advanced Mathematics, "Proceedings of the International Conference on Complex Geometry and Related Fields", Vol. 39 (2007), 1-21. MR2338616 (2008h:17012)

[BH] X. Bai and N. Hu, Two-parameter quantum groups of exceptional type E-series and convex PBW-type basis, Algebra Colloq. 15 (2008), 619-636. MR2451995 (2009g:17015)

[BW1] G. Benkart and S. Witherspoon, Two-parameter quantum groups and Drinfel'd doubles, Alg. Rep. Theory 7 (2004), 261-286. MR2070408 (2005g:17028)

[BW2] G. Benkart and S. Witherspoon, Representations of two-parameter quantum groups and Schur-Weyl duality, Hopf algebras, 62-92, Lecture Notes in Pure and Appl. Math., 237, Dekker, New York, 2004. MR2051731 (2005g:17027)

[BW3] G. Benkart and S. Witherspoon, Restricted two-parameter quantum groups, Fields Institute Communications, "Representations of Finite Dimensional Algebras and Related Topics in Lie Theory and Geometry", Vol. 40 (2004), 293-318. MR2057401(2005b:17027)

[Dr] V. G. Drinfel'd, A new realization of Yangians and quantized affine algebras, Soviet Math. Dokl. 36 (1988), 212-216. MR914215 (88j:17020)

[FJ] I. Frenkel and N. Jing, Vertex representations of quantum affine algebras, Proc. Nat'l. Acad. Sci. USA. 85 (1998), 9373-9377. MR973376 (90e:17028)

[FK] I. Frenkel and V. Kac, Basic representations of affine Lie algebras and dual resonance models, Invent. Math. 62 (1980), 23-66. MR595581 (84f:17004)

[FJW1] I. Frenkel, N. Jing, and W. Wang, Vertex representations via finite groups and the McKay correspondence, Int'l. Math. Res. Notices 4 (2000), 196-222. MR.1747618 (2001c:17042)

[FJW2] I. Frenkel, N. Jing, and W. Wang, Quantum vertex representations via finite groups and the McKay correspondence, Commun. Math. Phys., 211 (2000), 365-393. MR.1754520 (2002d:17013)

[GKV] V. Ginzburg, M. Kapranov, and E. Vasserot, Langlands reciprocity for algebraic surfaces, Math. Res. Lett. 2 (1995), 147-160. MR1324698 (96f:11086)

[HRZ] N. Hu, M. Rosso, and H. Zhang, Two-parameter Quantum Affine Algebra $U_{r, s}\left(\widehat{\mathfrak{s l}}_{n}\right)$, Drinfeld Realization and Quantum Affine Lyndon Basis, Commun. Math. Phys., 278 (2008), 453-486. MR2372766 (2009b:17033)

[HZ] N. Hu and H. Zhang, Vertex Representations of Two-parameter Quantum Affine Algebras $U_{r, s}(\widehat{\mathfrak{g}}):$ The Simply Laced Cases, Preprint (2006)

[J1] N. Jing, Twisted vertex representations of quantum affine algebras, Invent. Math. 102 (1990), 663-690. MR1074490 (92a:17019)

[J2] N. Jing, On Drinfel'd realization of quantum affine algebras, Ohio State Univ. Math. Res. Inst. Publ. de Gruyter, Berlin, 7 (1998), 195-206. MR1650669 (99j:17021)

[J3] N. Jing, Quantum Kac-Moody algebras and vertex representations, Lett. Math. Phys. 44 (1998), 261-271. MR1627867 (99j:17043)

[J4] N. Jing, Vertex representations and McKay correspondence. Algebra Colloq. 11 (2004), no. 1, 53-70. MR.2058964(2005e:17048)

[K] V. G. Kac, Infinite Dimensional Lie Algebras, 3rd edition, Cambridge Univ. Press, 1990. MR:1104219 (92k:17038)

[M] I. G. Macdonald, Symmetric functions and Hall polynomials, 2nd ed. Oxford: Clarendon Press, 1995. MR.1354144 (96h:05207) 
[Mc] J. McKay, Graphs, singularities and finite groups, Proc. Sympos. Pure Math. 37 (1980), 183-186. MR604577 (82e:20014)

[N] H. Nakajima, Quiver varieties and finite-dimensional representations of quantum affine algebras, J. Amer. Math. Soc. 14 (2001), no. 1, 145-238. MR1808477 (2002i:17023)

[R] N. Reshetikhin, Multiparameter quantum groups and twisted quasitriangular Hopf algebras, Lett. Math. Phys. 20 (1990), no. 4, 331-335. MR.1077966 (91k:17012)

[S] Y. Saito, Quantum toroidal algebras and their vertex representations, Publ. Res. Inst. Math. Sci. 34 (1998), no. 2, 155-177. MR1617066 (99d:17022)

[T] M. Takeuchi, A two-parameter quantization of $G L(n)$, Proc. Japan Acad. 66 Ser. A (1990), 112-114. MR1065785 (92f:16049)

[VV] V. Varagnolo and E. Vasserot, Double-loop algebras and the Fock space, Invent. Math. 133 (1998), 133-159. MR:1626481 (99g:17035)

[W] W. Wang, Equivariant K-theory, wreath products and Heisenberg algebra, Duke Math. J. 103 (2000), 1-23. MR.1758236 (2001b:19005)

[Z] H. Zhang, Drinfeld realizations, quantum affine Lyndon bases and vertex representations of two-parameter quantum affine algebras, Ph.D. thesis, ECNU, Shanghai, China, 2007.

School of Sciences, South China University of Technology, Guangzhou 510640, PeoPle's Republic of China - and - Department of Mathematics, North Carolina State University, Raleigh, North Carolina 27695

E-mail address: jing@math.ncsu.edu

Department of Mathematics, Shanghai University, Shanghai 200444, People's RepubLIC OF CHINA

E-mail address: hlzhangmath@shu.edu.cn 